\title{
Exhaustive Fluid Vacation Model with Positive Fluid Rate during Service ${ }^{\frac{1}{3}}$
}

\author{
Gábor Horváth ${ }^{\mathrm{a}, \mathrm{b}}$, Miklós Telek ${ }^{\mathrm{a}, \mathrm{b}}$ \\ ${ }^{a}$ MTA-BME Information Systems Research Group, Budapest, Hungary \\ ${ }^{b}$ Budapest University of Technology and Economics, Department of Networked Systems and Services, Budapest, Hungary
}

\begin{abstract}
Using an elegant transform domain iterative operator approach exhaustive fluid vacation models with strictly negative fluid rate during service has been analyzed, recently. Unfortunately, the potential presence of positive fluid rate during service (when the fluid input rate is larger than the fluid service rate) inhibits the use of all previously applied methodologies and makes the extension of the analysis approaches of discrete vacation and polling models toward fluid vacation and polling models rather difficult.

Based on the level crossing analysis of Markov fluid models the paper introduces an analysis approach which is applicable for the stationary analysis of the fluid level distribution and its moments. In the course of the analysis compact new matrix exponential expressions are obtained for the distribution of fluid level during a busy period starting from a given positive fluid level and by exploiting the relations of upward and downward measures of fluid processes several matrix transformations are applied to avoid Kronecker expansion matrix multiplications.
\end{abstract}

Finally, the obtained fluid level distribution is related with previous results of fluid vacation models.

Keywords: Fluid vacation model, Exhaustive discipline, Markov fluid queue, level crossing, busy period.

\section{Introduction}

Vacation and polling models with discrete (integer) customers have been studied $[1,2]$ and applied on a wide range of application fields of stochastic modeling for long time $[3,4]$. An interesting property of the vacation models is the stochastic decomposition property [5], which means that the steady-state number of customers in the system can be decomposed to the sum of two independent random variables, the steadystate number of customers in the corresponding queue and the steady-state number of customers presents in the system at arbitrary epoch in the vacation period.

With the evolution of fluid queueing models $[6,7,8,9]$ and their use in applied modeling [10] the question of the fluid counterpart of vacation and polling models has arisen. A first step toward this direction is by Czerniak and Yechiali [11] whose model is rather limited with respect to the stochastic evolution of the considered process: the load and the fluid service rate of stations are constant and the only stochastic ingredient of the model is the switchover time.

A next step towards general fluid polling models was the analysis of fluid vacation models in which the fluid source is modulated by a background Markov chain, the service discipline is gated and the vacation (switchover) time is generally distributed [12]. A consecutive work evaluates the same model with exhaustive discipline under the assumption that fluid rate is strictly negative during the service period [13]. The analysis of these two fluid vacation models is based on an analysis approach which is inherited from discrete vacation model analysis. This analysis method starts with the transform domain description of the buffer content at service start epoch as a function of the buffer content at vacation start epoch and the same analysis at

\footnotetext{
This work was supported by the Hungarian research project OTKA K101150, and by the János Bolyai Research Scholarship of the Hungarian Academy of Sciences. The author thank the careful reviews.

Email addresses: ghorvath@hit.bme.hu (Gábor Horváth), telek@hit.bme.hu (Miklós Telek)
} 
vacation start as a function of fluid level at service start. The solution of the obtained set of transform function equations give the stationary distributions at these (vacation and service start) embedded time points. The final step of the analysis is the evaluation of the time stationary distribution of the fluid content based on the embedded ones.

The seemingly minor technical detail, strictly negative fluid rate during service, has a crucial role in the applicability of the outlined analysis approach. Our goal in this paper is to relax this small technical restriction. Unfortunately, the potential presence of positive fluid rate during service requires the introduction of a completely different analysis approach. Our approach is based on the analysis of level crossings during a vacation+service cycle. The analysis of level crossings in stochastic fluid models is introduced by the matrix analytic method community $[8,9,14]$. We apply several existing level crossing results from those works, but the analysis of fluid vacation model requires the introduction and analysis of previously not considered level crossing measures. For example, we obtained matrix exponential expressions for the mean number of level crossings during a busy period starting from a positive fluid level. A submitted extended version of [13] discusses the spacial case of fluid vacation models with exhaustive discipline and phase type distributed vacation time. It presents an analysis method based on the Kronecker sum of the background Markov chain generator and the phase type generator, but that methodology is not applicable when the vacation time is not phase type distributed.

Our large term research goal is the analysis of fluid polling models. The current paper is a small step towards this direction, where we still focus on vacation models (single station polling system), but relax an impractical restriction on the fluid rates. The rest of the paper is organized as follows. The next section presents the considered model behavior and modeling assumptions. The model behavior during service period is investigated in Section 3. The Laplace transform description of the stationary distribution of the fluid level and its mean are obtained in Section 4 and 5, respectively. A small numerical example concludes the paper in Section 6.

\section{Model description}

We consider a fluid vacation model with Markov modulated load and exhaustive discipline. The model has an infinite fluid buffer.

The input fluid flow of the buffer is determined by a modulating CTMC $(\Omega(t)$ for $t \geq 0)$ with state space $\mathcal{S}=\{1, \ldots, N\}$ and generator $\mathbf{Q}$. When this Markov chain is in state $j(\Omega(t)=j)$ then fluid flows to the buffer at rate $r_{j}$ for $j \in\{1, \ldots, N\}$. We define the diagonal matrix $\mathbf{R}=\operatorname{diag}\left\langle r_{1}, \ldots, r_{N}\right\rangle$. During the service period the server removes fluid from the buffer at finite rate $d>0$. Consequently, when the overall Markov chain is in state $j(\Omega(t)=j)$ then the fluid level of the buffer during the service period changes at rate $r_{j}-d$, otherwise during the vacation periods it changes at rate $r_{j}$, because there is no service.

It is an important restriction in the current work that we consider only non-zero fluid rates, that is, $r_{j}>0$ and $r_{j}-d \neq 0$ for $j \in\{1, \ldots, N\}$. The difficulty of relaxing these restrictions is similar to the generalization of Markov fluid models without zero rates to the ones with possible zero fluid rates, but the notations used for the analysis of the current problem without zero fluid rates are rather complicated and we omit the case with zero fluid rate which requires a more complex set of notations.

We subdivide the set of states to $\mathcal{S}^{+}$and $\mathcal{S}^{-}$according to the sign of $r_{i}-d$. Without loss of generality we assume that the indexes of the states in $\mathcal{S}^{+}$are lower than the ones in $\mathcal{S}^{-}$. Accordingly, matrices $\mathbf{Q}$ and $\mathbf{R}$ are partitioned as

$$
\mathbf{Q}=\left[\begin{array}{ll}
\mathbf{Q}^{++} & \mathbf{Q}^{+-} \\
\mathbf{Q}^{-+} & \mathbf{Q}^{--}
\end{array}\right], \quad \mathbf{R}=\left[\begin{array}{cc}
\mathbf{R}^{+} & \mathbf{0} \\
\mathbf{0} & \mathbf{R}^{-}
\end{array}\right]
$$

When the fluid rate is strictly negative during service, which is the assumption in [13], then $\mathcal{S}^{+}=\emptyset$, and all below discussed analytical problems associated with the $\mathcal{S}^{+}, \mathcal{S}^{-}$division of the states are avoided.

In the vacation model the length of the service period is determined by the applied discipline. In this work we consider the exhaustive discipline. Under exhaustive discipline the fluid is removed during the service period until the buffer becomes empty. Each time the buffer becomes empty the server takes a vacation 
period. During vacation periods there is no service thus the fluid level of the buffer is increasing by the actual fluid rates. The consecutive vacation times are independent and identically distributed (i.i.d.). The random variable of the vacation time, its probability distribution function (pdf), its Laplace transform $(\mathrm{LT})$, its $i$ th moment and its squared coefficient of variation are denoted by $\sigma, \sigma(t)=\frac{\mathrm{d}}{\mathrm{d} t} \operatorname{Pr}(\sigma<t)$, $\sigma^{*}(s)=E\left(e^{-s \sigma}\right), E\left(\sigma^{i}\right)$ and $c_{\sigma}^{2}=\frac{E\left(\sigma^{2}\right)}{E^{2}(\sigma)}-1$, respectively. We define the cycle time (or simple cycle) as the time between just after the starts of two consecutive service periods. In the sequel we apply the notation $\sigma^{*}(s)=E\left(e^{-s \sigma}\right)=\int_{t} e^{-s t} \sigma(t) \mathrm{d} t$ not only for scalar values but for square matrices as well. E.g. for matrix $\mathbf{X}$ we have $\sigma^{*}(\mathbf{X})=E\left(e^{-\mathbf{X} \sigma}\right)=\int_{t} e^{-\mathbf{X} t} \sigma(t) \mathrm{d} t$.

We set the following assumptions on the fluid vacation model:

- A.1 The generator matrix $\mathbf{Q}$ of the modulating CTMC is finite and irreducible.

- A.2 The fluid rates are positive and finite, i.e. $r_{j}>0$ for $j \in\{1, \ldots, N\}$.

- A.3 The fluid is removed from the buffer according to the FCFS discipline.

Let $\pi$ be the stationary probability vector of the modulating Markov chain. Due to assumption A.1 the equations

$$
\pi \mathbf{Q}=0, \quad \pi \mathbb{1}=1
$$

uniquely determine $\pi$, where $\mathbb{1}$ is the $N \times 1$ column vector of ones. The stationary fluid flow rate, $\lambda$, and and the utilization $\rho$, are given as

$$
\lambda=\pi \mathbf{R} 1, \quad \rho=\frac{\lambda}{d},
$$

respectively. The necessary and sufficient condition of the stability of the fluid vacation model (since the amount of fluid served during a service period is unlimited) is that the mean fluid arrival rate $\lambda$ is less than $d$, which is equivalent with $\rho<1$.

For the $i, j$-th element of the matrix $\mathbf{X}$ the notation $\mathbf{X}_{i, j}$ is used. Similarly, $x_{j}$ denotes the $j$-th element of vector $x$. When $\mathbf{X}^{*}(s), \operatorname{Re}(s) \geq 0$ is a matrix LT (the elements of the matrix are LT of random variables), $\mathbf{X}^{(k)}$ denotes its $k$-th $(k \geq 1)$ derivative at $s=0$, i.e., $\mathbf{X}^{(k)}=\left.\frac{d^{k}}{d s^{k}} \mathbf{X}^{*}(s)\right|_{s=0}$ and $\mathbf{X}$ denotes its value at $s=0$, i.e., $\mathbf{X}=\mathbf{X}^{*}(0)$. Similar notations are applied for vector LT and scalar LT.

\subsection{System behavior during the vacation period}

During the vacation period all the fluid rates are positive, thus the fluid arrival process resembles a reward model [15]. In this section we consider the accumulated fluid during time $t \geq 0$. More precisely we derive the matrix LT of the fluid flowing into the buffer as a function of time, where the rows and columns of the matrix LT represent the initial and the final states of the modulating Markov chain. Let $\mathcal{Y}(t) \in \mathbb{R}^{+}$ be the accumulated fluid arrived at the buffer until time $t$, and let $\mathbf{A}(t, y)$ be the transition density matrix composed by elements $\mathbf{A}_{j, k}(t, y)=\frac{\partial}{\partial y} \operatorname{Pr}(\Omega(t)=k, \mathcal{Y}(t)<y \mid \Omega(0)=j, \mathcal{Y}(0)=0)$. The differential equations describing the evolution of $\mathbf{A}(t, x)$ are [15]

$$
\frac{\partial}{\partial t} \mathbf{A}(t, x)+\frac{\partial}{\partial x} \mathbf{A}(t, x) \mathbf{R}=\mathbf{A}(t, x) \mathbf{Q}
$$

with initial conditions

$$
\mathbf{A}(0, x)=\delta(x) \mathbf{I} \text { and } \mathbf{A}(t, 0)=\mathbf{0}, \quad \forall t>0,
$$

where $\delta(x)$ denotes the Kronecker delta and $\mathbf{I}$ is the identity matrix. Based on these differential equations the LST of $\mathbf{A}(t, x)$ with respect to $x$ is matrix exponential, $\mathbf{A}^{*}(t, s)=\int_{x=0}^{\infty} \mathbf{A}(t, x) e^{-s x} \mathrm{~d} x=e^{(\mathbf{Q}-s \mathbf{R}) t}$. 


\section{The behavior of the vacation queue during the service periods}

During the service periods the fluid level increases in states $\mathcal{S}^{+}$, and it decreases in states $\mathcal{S}^{-}$. The system behaves like a fluid model. Several solution techniques exist for the analysis of fluid models. In this paper we follow the matrix-analytic approach.

We start the section by recalling the results available for two important quantities related to fluid models:

- the mean number of level crossings before hitting a boundary,

- and the phase transition probabilities related to hitting boundaries.

Then, Section 3.3 provides a new contribution, the mean number of level crossings with non-zero initial level, that is needed later for the queue length analysis of the vacation queue.

\subsection{Matrix-analytic solution of fluid models}

The solution of fluid models where the fluid rates are either +1 or -1 , also called as canonical fluid models are especially simply to analyze $[16,9]$. Canonical fluid models are fully characterized by the generator of the background process $\tilde{\mathbf{Q}}$ and the number of states with positive rates $\left|\mathcal{S}^{+}\right|$. In our case the fluid model corresponding to the service periods of the vacation queue given by matrices $(\mathbf{Q}, \mathbf{R}-d \mathbf{I})$ is transformed to a canonical fluid model as

$$
\tilde{\mathbf{Q}}=|\mathbf{R}-d \mathbf{I}|^{-1} \mathbf{Q}, \quad \tilde{\mathbf{R}}=\left[\begin{array}{cc}
\mathbf{I} & \mathbf{0} \\
\mathbf{0} & -\mathbf{I}
\end{array}\right],
$$

where $\tilde{\mathbf{R}}$ is fully defined by $\left|\mathcal{S}^{+}\right|$.

Several important quantities of the original fluid model can either be easily derived from the ones of the transformed model or are exactly the same as the ones of the transformed model (like the expected number of crossings of various fluid levels).

There are characteristic matrices that play fundamental roles in the analysis of the fluid models. Their definitions and probabilistic interpretations are enumerated below.

- Matrix $\boldsymbol{\Psi}$. Entry $\boldsymbol{\Psi}_{i, j}, i \in \mathcal{S}^{+}, j \in \mathcal{S}^{-}$is the probability that the background process is in state $j$ when the fluid level returns to 0 given that it was in state $i$ when the busy period (a non-empty period of the fluid queue) was initiated. If $\rho<1$ then the fluid level surely returns to 0 and $\boldsymbol{\Psi} \mathbb{1}=\mathbb{1}$. Matrix $\boldsymbol{\Psi}$ is the minimal non-negative solution to the non-symmetric algebraic Riccati equation (NARE)

$$
\mathbf{\Psi} \tilde{\mathbf{Q}}^{-+} \boldsymbol{\Psi}+\boldsymbol{\Psi} \tilde{\mathbf{Q}}^{--}+\tilde{\mathbf{Q}}^{++} \mathbf{\Psi}+\tilde{\mathbf{Q}}^{+-}=\mathbf{0} .
$$

- Matrix K. Entry $e^{\mathbf{K} x}{ }_{i, j}$ is the expected number of crossings of fluid level $x$ in phase $j \in \mathcal{S}^{+}$starting from level 0 and phase $i \in \mathcal{S}^{+}$, before returning to level 0 . If $\rho<1$ all eigenvalues of matrix $\mathbf{K}$ have negative real parts (thus it is full rank and invertible) and can be expressed from $\boldsymbol{\Psi}$ as

$$
\mathbf{K}=\tilde{\mathbf{Q}}^{++}+\mathbf{\Psi} \tilde{\mathbf{Q}}^{-+}
$$

Starting from level 0 the expected number of level crossings at level $x$ for both positive and negative states is given by $N^{+} \times N$ matrix $\mathbf{N}(x)$, obtained by

$$
\mathbf{N}(x)=e^{\mathbf{K} x}\left[\begin{array}{ll}
\mathbf{I} & \mathbf{\Psi}
\end{array}\right] .
$$

- Matrix $\mathbf{U}$ is the $N^{-} \times N^{-}$generator of a CTMC called "downward records", which characterizes the background process $\Omega(t)$ observed in $\mathcal{S}^{-}$only. If $\rho<1$ matrix $\mathbf{U}$ is a proper generator such that $\mathbf{U} \mathbb{1}=0$ and $e^{\mathbf{U} x} \mathbb{1}=\mathbb{1}$. It can be expressed from matrix $\boldsymbol{\Psi}$ as

$$
\mathbf{U}=\tilde{\mathbf{Q}}^{--}+\tilde{\mathbf{Q}}^{-+} \mathbf{\Psi} .
$$


Matrix $\mathbf{U}$ has an important role in the transient analysis of fluid models as well. Entry $e^{\mathbf{U} x}{ }_{i, j}$ is the probability that the background process is in state $j \in \mathcal{S}^{-}$when level 0 is hit for the first time, starting from phase $i \in \mathcal{S}^{-}$and level $x>0$. More formally,

$$
e^{\mathbf{U} x}{ }_{i, j}=\operatorname{Pr}\left(\Omega\left(\gamma^{(0)}\right)=j \mid \mathcal{X}(0)=x, \Omega(0)=i\right), \quad x>0,
$$

where $\gamma^{(0)}$ is the first time when level 0 is hit, $\gamma^{(0)}=\min \{t: \mathcal{X}(t)=0\}$.

Note that both the expected number of level crossings $\left(e^{\mathbf{K} x}\right)$ and the phase transition probability related matrices during the busy period $\left(\Psi\right.$ and $\left.e^{\mathbf{U} x}\right)$, are the same for the canonical fluid model given by $(\tilde{\mathbf{Q}}, \tilde{\mathbf{R}})$ and the original one given by $(\mathbf{Q}, \mathbf{R}-d \mathbf{I})$.

\subsection{Solution of fluid models with finite buffer}

If fluid buffer is finite with size $x$ four different characterizing hitting probabilities can be defined depending on the initial and the final fluid levels, as

$$
\begin{aligned}
& \Psi^{(x)}{ }_{i, j}=\operatorname{Pr}\left(\Omega\left(\gamma^{(0)}\right)=j, \gamma^{(0)}<\gamma^{(x)} \mid \Omega(0)=i, \mathcal{X}(0)=0\right), \text { for } i \in \mathcal{S}^{+}, j \in \mathcal{S}^{-}, \\
& \boldsymbol{\Lambda}^{(x)}{ }_{i, j}=\operatorname{Pr}\left(\Omega\left(\gamma^{(x)}\right)=j, \gamma^{(x)}<\gamma^{(0)} \mid \Omega(0)=i, \mathcal{X}(0)=0\right), \text { for } i \in \mathcal{S}^{+}, j \in \mathcal{S}^{+}, \\
& \hat{\boldsymbol{\Psi}}^{(x)}{ }_{i, j}=\operatorname{Pr}\left(\Omega\left(\gamma^{(x)}\right)=j, \gamma^{(x)}<\gamma^{(0)} \mid \Omega(0)=i, \mathcal{X}(0)=x\right), \text { for } i \in \mathcal{S}^{-}, j \in \mathcal{S}^{+}, \\
& \hat{\boldsymbol{\Lambda}}^{(x)}{ }_{i, j}=\operatorname{Pr}\left(\Omega\left(\gamma^{(0)}\right)=j, \gamma^{(0)}<\gamma^{(x)} \mid \Omega(0)=i, \mathcal{X}(0)=x\right), \text { for } i \in \mathcal{S}^{-}, j \in \mathcal{S}^{-},
\end{aligned}
$$

where $\gamma^{(y)}$ is the first time when level $y$ is hit, $\gamma^{(y)}=\min \{t: \mathcal{X}(t)=y, t>0\}$.

To obtain these matrices, the analysis of the level-reversed process is also necessary. The fundamental matrices corresponding to the level-reverse process are derived by swapping the role of states $\mathcal{S}^{+}$and $\mathcal{S}^{-}$. Hence we have that $N^{-} \times N^{+}$matrix $\hat{\boldsymbol{\Psi}}$ is the solution to NARE

$$
\hat{\mathbf{\Psi}} \tilde{\mathbf{Q}}^{+-} \hat{\boldsymbol{\Psi}}+\hat{\mathbf{\Psi}} \tilde{\mathbf{Q}}^{++}+\tilde{\mathbf{Q}}^{--} \hat{\mathbf{\Psi}}+\tilde{\mathbf{Q}}^{-+}=\mathbf{0}
$$

and matrices $\hat{\mathbf{K}}$ and $\hat{\mathbf{U}}$ are obtained by

$$
\hat{\mathbf{K}}=\tilde{\mathbf{Q}}^{--}+\hat{\mathbf{\Psi}} \tilde{\mathbf{Q}}^{+-}, \quad \hat{\mathbf{U}}=\tilde{\mathbf{Q}}^{++}+\tilde{\mathbf{Q}}^{+-} \hat{\mathbf{\Psi}} .
$$

If $\rho<1$ then the level-reversed process (with infinite buffer) is a transient process, and its fluid level increases to infinity. Consequently, the fluid level does not return to 0 with probability one, that is $\hat{\Psi} \mathbb{1} \leq \mathbb{1}$, and by similar reasons $\hat{\mathbf{K}}$ has a zero eigenvalue, $\hat{\mathbf{U}}$ is a transient generator, that is $\hat{\mathbf{U}} \mathbb{1} \leq 0$ and $e^{\hat{\mathbf{U}} x} \mathbb{1} \leq \mathbb{1}$.

According to $[16,9]$, the hitting probabilities defined above are expressed as

$$
\left[\begin{array}{ll}
\boldsymbol{\Lambda}^{(x)} & \boldsymbol{\Psi}^{(x)} \\
\hat{\mathbf{\Psi}}^{(x)} & \hat{\boldsymbol{\Lambda}}^{(x)}
\end{array}\right]=\left[\begin{array}{cc}
e^{\hat{\mathbf{U}} x} & \boldsymbol{\Psi} \\
\hat{\mathbf{\Psi}} & e^{\mathbf{U} x}
\end{array}\right] \cdot\left[\begin{array}{cc}
\mathbf{I} & \boldsymbol{\Psi} e^{\mathbf{U} x} \\
\hat{\mathbf{\Psi}} e^{\hat{\mathbf{U}} x} & \mathbf{I}
\end{array}\right]^{-1}
$$

Matrices $\boldsymbol{\Lambda}^{(x)}, \Psi^{(x)}, \hat{\Psi}^{(x)}$ and $\hat{\Lambda}^{(x)}$ are associated with a finite buffer fluid queue. Starting from level 0 one of the boundaries is reached with probability one, from which $\Lambda^{(x)} \mathbb{1}+\Psi^{(x)} \mathbb{1}=\mathbb{1}$ and the same holds starting from level $x, \hat{\mathbf{\Psi}}^{(x)} \mathbb{1}+\hat{\mathbf{\Lambda}}^{(x)} \mathbb{1}=\mathbb{1}$.

Similar to matrix $\mathbf{N}(x)$ defined in Section 3.1, we define matrix $\mathbf{N}^{(x)}(w, y)$ as follows. For $i, j \in \mathcal{S}$ let $\mathbf{N}_{i, j}^{(x)}(w, y)$ be the expected number of level crossing of level $y$ in state $j$ starting from level $w$ and state $i$ before having an empty buffer $(\mathcal{X}=0)$ or a full buffer $(\mathcal{X}=x)$.

For $i \in \mathcal{S}^{-}$and $y>0$ we have $\mathbf{N}_{i, j}^{(x)}(0, y)=0$ and for similarly for $i \in \mathcal{S}^{+}$and $y<x$ we have $\mathbf{N}_{i, j}^{(x)}(x, y)=0$. The matrices containing the non-trivial elements are $\mathbf{N}_{+}^{(x)}(0, y)$ of size $\left|\mathcal{S}^{+}\right| \times|\mathcal{S}|$, and $\mathbf{N}_{-}^{(x)}(x, y)$ of size $\left|\mathcal{S}^{-}\right| \times|\mathcal{S}|$ and satisfy

$$
\left[\begin{array}{l}
\mathbf{N}_{+}^{(x)}(0, y) \\
\mathbf{N}_{-}^{(x)}(x, y)
\end{array}\right]=\left[\begin{array}{cc}
\mathbf{I} & e^{\mathbf{K} x} \mathbf{\Psi} \\
e^{\hat{\mathbf{K}} x} \hat{\mathbf{\Psi}} & \mathbf{I}
\end{array}\right]^{-1} \cdot\left[\begin{array}{cc}
e^{\mathbf{K} y} & \mathbf{0} \\
\mathbf{0} & e^{\hat{\mathbf{K}}(x-y)}
\end{array}\right] \cdot\left[\begin{array}{cc}
\mathbf{I} & \boldsymbol{\Psi} \\
\hat{\mathbf{\Psi}} & \mathbf{I}
\end{array}\right] .
$$




\subsection{Level crossings during the busy period if the initial fluid level is $x$}

In this section we investigate the fluid level distribution during a busy period of an infinite fluid queue starting from level $x$, by evaluating the level crossing events at various fluid levels. For $i, j \in \mathcal{S}$ let $\mathbf{M}_{i, j}(x, y)$ be the expected number of crossings of level $y$ in state $j$ starting from state $i$ and fluid level $x$ before having an empty buffer. That is

$$
\mathbf{M}_{i, j}(x, y)=E\left(\int_{t} \mathcal{I}\left\{t<\gamma^{(0)}, \Omega(t)=j, \mathcal{X}(t)=y\right\} \mathrm{d} t \mid \Omega(0)=i, \mathcal{X}(0)=x\right),
$$

where $\mathcal{I}\{\bullet\}$ is the indicator of event $\bullet$. In the rest of this section we derive closed form expressions for $\mathbf{M}(x, y)$.

The state of the fluid model at consecutive crossings of level $x$ forms a transient DTMC with generator $\left[\begin{array}{cc}\mathbf{0} & \mathbf{\Psi} \\ \hat{\mathbf{\Psi}}^{(x)} & \mathbf{0}\end{array}\right]$, where $\boldsymbol{\Psi} \mathbb{1}=\mathbb{1}$ and $\hat{\mathbf{\Psi}}^{(x)} \mathbb{1} \leq \mathbb{1}$ reflects the fact that the busy period can terminate only after crossing level $x$ with a negative rate. The expected number of crossings of level $x$ in various states is given by

$$
\mathbf{B}(x)=\left[\begin{array}{ll}
\mathbf{B}^{++}(x) & \mathbf{B}^{+-}(x) \\
\mathbf{B}^{-+}(x) & \mathbf{B}^{--}(x)
\end{array}\right]=\left(\mathbf{I}-\left[\begin{array}{cc}
\mathbf{0} & \mathbf{\Psi} \\
\hat{\mathbf{\Psi}}^{(x)} & \mathbf{0}
\end{array}\right]\right)^{-1}=\left[\begin{array}{cc}
\left(\mathbf{I}-\mathbf{\Psi} \hat{\mathbf{\Psi}}^{(x)}\right)^{-1} & \left(\mathbf{I}-\mathbf{\Psi} \hat{\mathbf{\Psi}}^{(x)}\right)^{-1} \mathbf{\Psi} \\
\left(\mathbf{I}-\hat{\mathbf{\Psi}}^{(x)} \mathbf{\Psi}\right)^{-1} \hat{\mathbf{\Psi}}^{(x)} & \left(\mathbf{I}-\hat{\mathbf{\Psi}}^{(x)} \mathbf{\Psi}\right)^{-1}
\end{array}\right] .
$$

The mean number of crossings of level $y>x$ can be computed relative to the mean number of crossings of level $x$ based on the results available for infinite fluid buffers, see (9). Thus, for $y>x$ we have

$$
\mathbf{M}(x, y)=\left[\begin{array}{l}
\mathbf{B}^{++}(x) \\
\mathbf{B}^{-+}(x)
\end{array}\right] e^{\mathbf{K}(y-x)}\left[\begin{array}{ll}
\mathbf{I} & \mathbf{\Psi}
\end{array}\right] .
$$

Similarly, the mean number of crossings of level $0<y<x$ is computed relative to the number of crossings of level $x$ based on the results available for finite fluid buffers, yielding

$$
\begin{aligned}
\mathbf{M}(x, y) & =\left[\begin{array}{l}
\mathbf{B}^{+-}(x) \\
\mathbf{B}^{--}(x)
\end{array}\right] \mathbf{N}_{-}^{(x)}(x, y) \\
& =\left[\begin{array}{l}
\mathbf{B}^{+-}(x) \\
\mathbf{B}^{--}(x)
\end{array}\right]\left(\mathbf{I}-e^{\hat{\mathbf{K}} x} \hat{\mathbf{\Psi}} e^{\mathbf{K} x} \mathbf{\Psi}\right)^{-1}\left(-e^{\hat{\mathbf{K}} x} \hat{\mathbf{\Psi}} e^{\mathbf{K} y}\left[\begin{array}{ll}
\mathbf{I} & \mathbf{\Psi}
\end{array}\right]+e^{\hat{\mathbf{K}}(x-y)}\left[\begin{array}{ll}
\hat{\mathbf{\Psi}} & \mathbf{I}
\end{array}\right]\right),
\end{aligned}
$$

where we utilized that from (19) it is possible to express $\mathbf{N}_{-}^{(x)}(x, y)=\left[\begin{array}{lll}\mathbf{N}_{-+}^{(x)}(x, y) & \mathbf{N}_{--}^{(x)}(x, y)\end{array}\right]$ as

$$
\begin{aligned}
& \mathbf{N}_{-+}^{(x)}(x, y)=-e^{\hat{\mathbf{K}} x} \hat{\mathbf{\Psi}}\left(\mathbf{I}-e^{\mathbf{K} x} \mathbf{\Psi} e^{\hat{\mathbf{K}} x} \hat{\mathbf{\Psi}}\right)^{-1} e^{\mathbf{K} y}+\left(\mathbf{I}-e^{\hat{\mathbf{K}} x} \hat{\mathbf{\Psi}} e^{\mathbf{K} x} \mathbf{\Psi}\right)^{-1} e^{\hat{\mathbf{K}}(x-y)} \hat{\mathbf{\Psi}}, \\
& \mathbf{N}_{--}^{(x)}(x, y)=-e^{\hat{\mathbf{K}} x} \hat{\mathbf{\Psi}}\left(\mathbf{I}-e^{\mathbf{K} x} \mathbf{\Psi} e^{\hat{\mathbf{K}} x} \hat{\mathbf{\Psi}}\right)^{-1} e^{\mathbf{K} y} \mathbf{\Psi}+\left(\mathbf{I}-e^{\hat{\mathbf{K}} x} \hat{\mathbf{\Psi}} e^{\mathbf{K} x} \mathbf{\Psi}\right)^{-1} e^{\hat{\mathbf{K}}(x-y)},
\end{aligned}
$$

and that $e^{\hat{\mathbf{K}} x} \hat{\mathbf{\Psi}}\left(\mathbf{I}-e^{\mathbf{K} x} \mathbf{\Psi} e^{\hat{\mathbf{K}} x} \hat{\mathbf{\Psi}}\right)^{-1}=\left(\mathbf{I}-e^{\hat{\mathbf{K}} x} \hat{\mathbf{\Psi}} e^{\mathbf{K} x} \mathbf{\Psi}\right)^{-1} e^{\hat{\mathbf{K}} x} \hat{\mathbf{\Psi}}$ holds.

Let us now express $\mathbf{M}(x, y)$ around fluid level $x$. From (22) we get

$$
\mathbf{M}(x, x+)=\left[\begin{array}{l}
\mathbf{B}^{++}(x) \\
\mathbf{B}^{-+}(x)
\end{array}\right]\left[\begin{array}{ll}
\mathbf{I} & \mathbf{\Psi}
\end{array}\right],
$$

which has a clear stochastic interpretation. Similarly, taking the limit $y \nearrow x$ in (23) leads to

$$
\mathbf{M}(x, x-)=\left[\begin{array}{l}
\mathbf{B}^{+-}(x) \\
\mathbf{B}^{--}(x)
\end{array}\right]\left(\mathbf{I}-e^{\hat{\mathbf{K}} x} \hat{\mathbf{\Psi}} e^{\mathbf{K} x} \mathbf{\Psi}\right)^{-1}\left(-e^{\hat{\mathbf{K}} x} \hat{\mathbf{\Psi}} e^{\mathbf{K} x}\left[\begin{array}{ll}
\mathbf{I} & \mathbf{\Psi}
\end{array}\right]+\left[\begin{array}{ll}
\hat{\mathbf{\Psi}} & \mathbf{I}
\end{array}\right]\right),
$$


however, by the stochastic interpretations of $\mathbf{M}(x, x-)$ and $\mathbf{B}(x)$ we also have that

$$
\mathbf{M}(x, x-)=\left[\begin{array}{l}
\mathbf{B}^{+-}(x) \\
\mathbf{B}^{--}(x)
\end{array}\right]\left[\begin{array}{ll}
\hat{\mathbf{\Psi}}^{(x)} & \mathbf{I}
\end{array}\right] .
$$

Equating (27) and (28) leads us to the following lemma.

Lemma 1. The following equality holds:

$$
(\mathbf{I}-\hat{\mathbf{\Psi}} \boldsymbol{\Psi})^{-1}=\left(\mathbf{I}-\hat{\mathbf{\Psi}}^{(x)} \mathbf{\Psi}\right)^{-1}\left(\mathbf{I}-e^{\hat{\mathbf{K}} x} \hat{\mathbf{\Psi}} e^{\mathbf{K} x} \mathbf{\Psi}\right)^{-1} .
$$

Proof. Based on the equality of (27) and (28) we get

$$
\hat{\mathbf{\Psi}}^{(x)}=\left(\mathbf{I}-e^{\hat{\mathbf{K}} x} \hat{\mathbf{\Psi}} e^{\mathbf{K} x} \mathbf{\Psi}\right)^{-1}\left(-e^{\hat{\mathbf{K}} x} \hat{\mathbf{\Psi}} e^{\mathbf{K} x}+\hat{\mathbf{\Psi}}\right) .
$$

Post-multiplying both sides by $\boldsymbol{\Psi}$ and subtracting from $\mathbf{I}$ gives

$$
\begin{aligned}
\mathbf{I}-\hat{\boldsymbol{\Psi}}^{(x)} \mathbf{\Psi} & =\mathbf{I}-\left(\mathbf{I}-e^{\hat{\mathbf{K}} x} \hat{\mathbf{\Psi}} e^{\mathbf{K} x} \mathbf{\Psi}\right)^{-1}\left(\mathbf{I}-e^{\hat{\mathbf{K}} x} \hat{\mathbf{\Psi}} e^{\mathbf{K} x} \mathbf{\Psi}-\mathbf{I}+\hat{\mathbf{\Psi}} \boldsymbol{\Psi}\right) \\
& =\left(\mathbf{I}-e^{\hat{\mathbf{K}} x} \hat{\mathbf{\Psi}} e^{\mathbf{K} x} \mathbf{\Psi}\right)^{-1}(\mathbf{I}-\hat{\mathbf{\Psi}} \boldsymbol{\Psi}),
\end{aligned}
$$

from which (29) follows.

Theorem 1. The density of the fluid level during the busy period given that the initial fluid level is $x$ is obtained by

$$
\mathbf{M}(x, y)=-\left[\begin{array}{c}
\mathbf{\Psi} \\
\mathbf{I}
\end{array}\right](\mathbf{I}-\hat{\mathbf{\Psi}} \mathbf{\Psi})^{-1} e^{\hat{\mathbf{K}} x} \hat{\mathbf{\Psi}} e^{\mathbf{K} y}\left[\begin{array}{ll}
\mathbf{I} & \boldsymbol{\Psi}
\end{array}\right]+\left[\begin{array}{c}
\mathbf{\Psi} \\
\mathbf{I}
\end{array}\right](\mathbf{I}-\hat{\mathbf{\Psi}} \mathbf{\Psi})^{-1} e^{\hat{\mathbf{K}}(x-y)}\left[\begin{array}{ll}
\hat{\mathbf{\Psi}} & \mathbf{I}
\end{array}\right]
$$

for $0<y<x$, and it is obtained by

$$
\mathbf{M}(x, y)=-\left[\begin{array}{c}
\mathbf{\Psi} \\
\mathbf{I}
\end{array}\right](\mathbf{I}-\hat{\mathbf{\Psi}} \mathbf{\Psi})^{-1} e^{\hat{\mathbf{K}} x} \hat{\mathbf{\Psi}} e^{\mathbf{K} y}\left[\begin{array}{ll}
\mathbf{I} & \mathbf{\Psi}
\end{array}\right]+\left[\begin{array}{c}
\mathbf{I} \\
\hat{\mathbf{\Psi}}
\end{array}\right](\mathbf{I}-\mathbf{\Psi} \hat{\mathbf{\Psi}})^{-1} e^{\mathbf{K}(y-x)}\left[\begin{array}{ll}
\mathbf{I} & \mathbf{\Psi}
\end{array}\right]
$$

for $y>x$.

Proof. The blocks of matrix $\mathbf{B}(x)$ given by (21) satisfy the following relations $\left(\mathbf{I}-\mathbf{\Psi} \hat{\mathbf{\Psi}}^{(x)}\right)^{-1} \mathbf{\Psi} \hat{\mathbf{\Psi}}^{(x)}+\mathbf{I}=$ $\left(\mathbf{I}-\mathbf{\Psi} \hat{\mathbf{\Psi}}^{(x)}\right)^{-1}$ and $\left(\mathbf{I}-\hat{\mathbf{\Psi}}^{(x)} \mathbf{\Psi}\right)^{-1} \hat{\mathbf{\Psi}}^{(x)} \mathbf{\Psi}+\mathbf{I}=\left(\mathbf{I}-\hat{\mathbf{\Psi}}^{(x)} \mathbf{\Psi}\right)^{-1}$.

First we prove (32). Starting from (23) and inserting the blocks of matrix $\mathbf{B}(x)$ based on (21) gives

$$
\begin{aligned}
\mathbf{M}(x, y) & =\left[\begin{array}{l}
\mathbf{B}^{+-}(x) \\
\mathbf{B}^{--}(x)
\end{array}\right]\left(\mathbf{I}-e^{\hat{\mathbf{K}} x} \hat{\mathbf{\Psi}} e^{\mathbf{K} x} \mathbf{\Psi}\right)^{-1}\left(-e^{\hat{\mathbf{K}} x} \hat{\mathbf{\Psi}} e^{\mathbf{K} y}\left[\begin{array}{ll}
\mathbf{I} & \mathbf{\Psi}
\end{array}\right]+e^{\hat{\mathbf{K}}(x-y)}\left[\begin{array}{ll}
\hat{\mathbf{\Psi}} & \mathbf{I}
\end{array}\right]\right) \\
& =\left[\begin{array}{c}
\mathbf{\Psi} \\
\mathbf{I}
\end{array}\right]\left(\mathbf{I}-\hat{\mathbf{\Psi}}^{(x)} \mathbf{\Psi}\right)^{-1}\left(\mathbf{I}-e^{\hat{\mathbf{K}} x} \hat{\mathbf{\Psi}} e^{\mathbf{K} x} \mathbf{\Psi}\right)^{-1}\left(-e^{\hat{\mathbf{K}} x} \hat{\mathbf{\Psi}} e^{\mathbf{K} y}\left[\begin{array}{ll}
\mathbf{I} & \mathbf{\Psi}
\end{array}\right]+e^{\hat{\mathbf{K}}(x-y)}\left[\begin{array}{ll}
\hat{\mathbf{\Psi}} & \mathbf{I}
\end{array}\right]\right),
\end{aligned}
$$

which, by applying Lemma 1 yields (32).

Let us now prove (33). In (22), $\mathbf{B}^{++}(x)$ and $\mathbf{B}^{-+}(x)$ are replaced by $\mathbf{B}^{+-}(x) \hat{\mathbf{\Psi}}^{(x)}+\mathbf{I}$ and $\mathbf{B}^{--}(x) \hat{\mathbf{\Psi}}^{(x)}$, respectively, based on (21). Applying algebraic manipulations similar to (34) yields

$$
\begin{aligned}
\mathbf{M}(x, y) & =\left[\begin{array}{l}
\mathbf{B}^{++}(x) \\
\mathbf{B}^{-+}(x)
\end{array}\right] e^{\mathbf{K}(y-x)}\left[\begin{array}{ll}
\mathbf{I} & \mathbf{\Psi}
\end{array}\right]=\left(\left[\begin{array}{l}
\mathbf{B}^{+-}(x) \\
\mathbf{B}^{--}(x)
\end{array}\right] \hat{\mathbf{\Psi}}^{(x)}+\left[\begin{array}{l}
\mathbf{I} \\
\mathbf{0}
\end{array}\right]\right) e^{\mathbf{K}(y-x)}\left[\begin{array}{ll}
\mathbf{I} & \mathbf{\Psi}
\end{array}\right] \\
& =\left(\left[\begin{array}{l}
\mathbf{\Psi} \\
\mathbf{I}
\end{array}\right]\left(\mathbf{I}-\hat{\mathbf{\Psi}}^{(x)} \mathbf{\Psi}\right)^{-1} \hat{\mathbf{\Psi}}^{(x)}+\left[\begin{array}{l}
\mathbf{I} \\
\mathbf{0}
\end{array}\right]\right) e^{\mathbf{K}(y-x)}\left[\begin{array}{ll}
\mathbf{I} & \mathbf{\Psi}
\end{array}\right] \\
& =\left(\left[\begin{array}{l}
\mathbf{\Psi} \\
\mathbf{I}
\end{array}\right](\mathbf{I}-\hat{\mathbf{\Psi}} \mathbf{\Psi})^{-1}\left(-e^{\hat{\mathbf{K}} x} \hat{\mathbf{\Psi}} e^{\mathbf{K} x}+\hat{\mathbf{\Psi}}\right)+\left[\begin{array}{l}
\mathbf{I} \\
\mathbf{0}
\end{array}\right]\right) e^{\mathbf{K}(y-x)}\left[\begin{array}{ll}
\mathbf{I} & \mathbf{\Psi}
\end{array}\right],
\end{aligned}
$$


where we utilized that $\left(\mathbf{I}-\hat{\mathbf{\Psi}}^{(x)} \mathbf{\Psi}\right)^{-1} \hat{\mathbf{\Psi}}^{(x)}=(\mathbf{I}-\hat{\mathbf{\Psi}} \mathbf{\Psi})^{-1}\left(-e^{\hat{\mathbf{K}} x} \hat{\mathbf{\Psi}} e^{\mathbf{K} x}+\hat{\mathbf{\Psi}}\right)$, which follows from (29) and (30). Manipulating (34) further leads to

$$
\begin{aligned}
\mathbf{M}(x, y) & =\left(\left[\begin{array}{l}
\mathbf{\Psi} \\
\mathbf{I}
\end{array}\right](\mathbf{I}-\hat{\mathbf{\Psi}} \mathbf{\Psi})^{-1}\left(-e^{\hat{\mathbf{K}} x} \hat{\mathbf{\Psi}} e^{\mathbf{K} x}+\hat{\mathbf{\Psi}}\right)+\left[\begin{array}{l}
\mathbf{I} \\
\mathbf{0}
\end{array}\right]\right) e^{\mathbf{K}(y-x)}\left[\begin{array}{ll}
\mathbf{I} & \mathbf{\Psi}
\end{array}\right] \\
& =\underbrace{\left(\left[\begin{array}{l}
\mathbf{\Psi} \\
\mathbf{I}
\end{array}\right](\mathbf{I}-\hat{\mathbf{\Psi}} \mathbf{\Psi})^{-1} \hat{\mathbf{\Psi}}+\left[\begin{array}{l}
\mathbf{I} \\
\mathbf{0}
\end{array}\right]\right)}_{\left[\begin{array}{c}
\mathbf{I} \\
\hat{\mathbf{\Psi}}
\end{array}\right](\mathbf{I}-\mathbf{\Psi} \hat{\mathbf{\Psi}})^{-1}} e^{\mathbf{K}(y-x)}\left[\begin{array}{ll}
\mathbf{I} & \mathbf{\Psi}
\end{array}\right]-\left[\begin{array}{c}
\mathbf{\Psi} \\
\mathbf{I}
\end{array}\right](\mathbf{I}-\hat{\mathbf{\Psi}} \mathbf{\Psi})^{-1} e^{\hat{\mathbf{K}} x} \hat{\mathbf{\Psi}} e^{\mathbf{K} y}\left[\begin{array}{ll}
\mathbf{I} & \mathbf{\Psi}
\end{array}\right]
\end{aligned}
$$

which establishes the theorem.

Notice that the first terms of (32) and (33) are the same, while the second terms are perfectly symmetric.

\section{The stationary distribution of the fluid level in the vacation queue}

Let us denote the joint stationary distribution of the fluid level and the background process by vector $q(y)$, with entries defined by $q(y)_{\ell}=\frac{\mathrm{d}}{\mathrm{d} y} \lim _{t \rightarrow \infty} P(\mathcal{X}(t)<y, \Omega(t)=\ell$. Exploiting that the density of the fluid level $y$ and the expected number of crossings of level $y$ in a stationary cycle are proportional and are related by the inverse of the fluid rates $[16], q(y)$ is given by

$$
q(y)=\frac{1}{c}(\underbrace{\int_{t=0}^{\infty} \sigma(t) \int_{x=0}^{t} \beta \mathbf{A}(x, y) \mathrm{d} x \mathrm{~d} t}_{L^{(v)}(y)}+\underbrace{\int_{t=0}^{\infty} \sigma(t) \int_{x=0}^{\infty} \beta \mathbf{A}(t, x) \mathbf{M}(x, y) \mathrm{d} x \mathrm{~d} t}_{L^{(s)}(y)} \cdot|\mathbf{R}-d \mathbf{I}|^{-1}),
$$

where size $N$ vector $\beta$ is the stationary phase distribution at the beginning of the vacation period, matrix $\mathbf{A}(t, x)$ characterizes the amount of arriving fluid during the vacation period of length $t$, and $\mathbf{M}_{i, j}(x, y)$ is the expected number of crossings of level $y$ in phase $j$ in the service period starting from level $x$ and phase $i$. Finally, the mean cycle time, $c$, which stands for normalization constant, is given by the next lemma.

Lemma 2. The normalization constant equals the mean cycle time, that is

$$
c=\frac{E(\sigma)}{1-\rho} .
$$

Proof. The long term mean fluid input and output rates of the vacation queue are identical, that is $c \lambda=(c-E(\sigma)) d$, where $c-E(\sigma)$ is the mean service time. The statement of the lemma follows using (3).

The remaining components for $q(y)$, namely the initial vector $(\beta)$, the fluid density during vacation $\left(L^{(v)}(y)\right)$ and the expected number of crossings during service $\left(L^{(s)}(y)\right)$ are provided by the following subsections.

\subsection{Important relations}

Lemma 3. The following relations hold between the fundamental matrices of the fluid queues:

$$
\begin{aligned}
& (\mathbf{I}-\hat{\mathbf{\Psi}} \mathbf{\Psi})^{-1} \hat{\mathbf{K}}=\mathbf{U}(\mathbf{I}-\hat{\mathbf{\Psi}} \mathbf{\Psi})^{-1}, \\
& (\mathbf{I}-\mathbf{\Psi} \hat{\mathbf{\Psi}})^{-1} \mathbf{K}=\hat{\mathbf{U}}(\mathbf{I}-\mathbf{\Psi} \hat{\Psi})^{-1} .
\end{aligned}
$$


Proof. Post- and pre-multiplying (39) by $\mathbf{I}-\hat{\Psi} \Psi$ and substituting the definitions of matrices $\hat{\mathbf{K}}$ and $\mathbf{U}$ based on (10) and (17) gives

$$
\tilde{\mathbf{Q}}^{--}+\tilde{\mathbf{Q}}^{-+} \boldsymbol{\Psi}-\hat{\Psi} \Psi \tilde{\mathbf{Q}}^{--}-\hat{\Psi} \Psi \tilde{\mathbf{Q}}^{-+} \boldsymbol{\Psi}=\tilde{\mathrm{Q}}^{--}+\hat{\boldsymbol{\Psi}} \tilde{\mathbf{Q}}^{+-}-\tilde{\mathbf{Q}}^{--} \hat{\Psi} \Psi-\hat{\Psi} \tilde{\mathbf{Q}}^{+-} \hat{\Psi} \Psi,
$$

that, after rearranging the terms leads to

$$
\underbrace{\left(\tilde{\mathbf{Q}}^{-+}+\tilde{\mathbf{Q}}^{--} \hat{\mathbf{\Psi}}+\hat{\mathbf{\Psi}} \tilde{\mathbf{Q}}^{+-} \hat{\mathbf{\Psi}}\right)}_{\hat{\mathbf{\Psi}} \tilde{\mathbf{Q}}^{++}} \boldsymbol{\Psi}=\hat{\boldsymbol{\Psi}} \underbrace{\left(\tilde{\mathbf{Q}}^{+-}+\boldsymbol{\Psi} \tilde{\mathbf{Q}}^{--}+\boldsymbol{\Psi} \tilde{\mathbf{Q}}^{-+} \boldsymbol{\Psi}\right)}_{\tilde{\mathbf{Q}}^{++} \boldsymbol{\Psi}} .
$$

Due to the Riccati equations for $\boldsymbol{\Psi}$ (see (7)) and for $\hat{\boldsymbol{\Psi}}$ (see (16)) both sides are equal to $\hat{\boldsymbol{\Psi}} \tilde{\mathbf{Q}}^{++} \boldsymbol{\Psi}$. Equation (40) can be proven similarly.

Lemma 4. The following identities hold between the parameters of the vacation queue:

$$
\begin{aligned}
& \mathbf{Q}\left[\begin{array}{c}
\mathbf{\Psi} \\
\mathbf{I}
\end{array}\right]=-(\mathbf{R}-d \mathbf{I})\left[\begin{array}{c}
\mathbf{\Psi} \\
\mathbf{I}
\end{array}\right] \mathbf{U}, \\
& \mathbf{Q}\left[\begin{array}{c}
\mathbf{I} \\
\hat{\mathbf{\Psi}}
\end{array}\right]=+(\mathbf{R}-d \mathbf{I})\left[\begin{array}{c}
\mathbf{I} \\
\hat{\mathbf{\Psi}}
\end{array}\right] \hat{\mathbf{U}} .
\end{aligned}
$$

Proof. Making use of the block-partitioned structure of $\mathbf{Q}$ (see (1)) and the relation between $\mathbf{Q}$ and $\tilde{\mathbf{Q}}$ given by (6) we get

$$
\mathbf{Q}\left[\begin{array}{c}
\mathbf{\Psi} \\
\mathbf{I}
\end{array}\right]=\left[\begin{array}{c}
\left(\mathbf{R}_{+}-d\right)\left(\tilde{\mathbf{Q}}_{++} \boldsymbol{\Psi}+\tilde{\mathbf{Q}}_{+-}\right) \\
-\left(\mathbf{R}_{-}-d\right)\left(\tilde{\mathbf{Q}}_{-+} \boldsymbol{\Psi}+\tilde{\mathbf{Q}}_{--}\right)
\end{array}\right]=\left[\begin{array}{c}
-\left(\mathbf{R}_{+}-d\right) \mathbf{\Psi} \mathbf{U} \\
-\left(\mathbf{R}_{-}-d\right) \mathbf{U}
\end{array}\right]=-(\mathbf{R}-d \mathbf{I})\left[\begin{array}{c}
\mathbf{\Psi} \\
\mathbf{I}
\end{array}\right] \mathbf{U},
$$

where we exploited that $\tilde{\mathbf{Q}}_{++} \boldsymbol{\Psi}+\tilde{\mathbf{Q}}_{+-}=-\boldsymbol{\Psi} \tilde{\mathbf{Q}}_{--}-\boldsymbol{\Psi} \tilde{\mathbf{Q}}_{-+} \boldsymbol{\Psi}=-\boldsymbol{\Psi} \mathbf{U}$ due to (7). Equation (44) can be proven similarly.

Corollary 1. From Lemma 3 and Lemma 4 we also have

$$
\begin{aligned}
& \mathbf{Q}\left[\begin{array}{c}
\mathbf{\Psi} \\
\mathbf{I}
\end{array}\right](\mathbf{I}-\hat{\mathbf{\Psi}} \mathbf{\Psi})^{-1}=-(\mathbf{R}-d \mathbf{I})\left[\begin{array}{c}
\mathbf{\Psi} \\
\mathbf{I}
\end{array}\right](\mathbf{I}-\hat{\mathbf{\Psi}} \mathbf{\Psi})^{-1} \hat{\mathbf{K}}, \\
& \mathbf{Q}\left[\begin{array}{c}
\mathbf{I} \\
\hat{\mathbf{\Psi}}
\end{array}\right](\mathbf{I}-\mathbf{\Psi} \hat{\mathbf{\Psi}})^{-1}=+(\mathbf{R}-d \mathbf{I})\left[\begin{array}{c}
\mathbf{I} \\
\hat{\mathbf{\Psi}}
\end{array}\right](\mathbf{I}-\mathbf{\Psi} \hat{\mathbf{\Psi}})^{-1} \mathbf{K} .
\end{aligned}
$$

Lemma 5. The following identities hold between the parameters of the vacation queue:

$$
\begin{aligned}
& \mathbf{K}^{i}\left[\begin{array}{ll}
\mathbf{I} & \mathbf{\Psi}
\end{array}\right]|\mathbf{R}-d \mathbf{I}|^{-1}=\left[\begin{array}{ll}
\mathbf{I} & \mathbf{\Psi}
\end{array}\right]|\mathbf{R}-d \mathbf{I}|^{-1}\left(\mathbf{Q}(\mathbf{R}-d \mathbf{I})^{-1}\right)^{i}, \\
& (-\hat{\mathbf{K}})^{i}\left[\begin{array}{ll}
\hat{\mathbf{\Psi}} & \mathbf{I}
\end{array}\right]|\mathbf{R}-d \mathbf{I}|^{-1}=\left[\begin{array}{ll}
\hat{\mathbf{\Psi}} & \mathbf{I}
\end{array}\right]|\mathbf{R}-d \mathbf{I}|^{-1}\left(\mathbf{Q}(\mathbf{R}-d \mathbf{I})^{-1}\right)^{i} .
\end{aligned}
$$

ProOF. For $i=1$ this lemma is the row-wise counterpart of Lemma 4, and can be proven similarly.

For $i>1$ the proof is based on induction. Assume it holds for $i-1$. For $i$ we have that

$$
\begin{aligned}
\mathbf{K}^{i-1}\left(\mathbf{K}\left[\begin{array}{ll}
\mathbf{I} & \mathbf{\Psi}
\end{array}\right]|\mathbf{R}-d \mathbf{I}|^{-1}\right) & =\mathbf{K}^{i-1}\left[\begin{array}{ll}
\mathbf{I} & \mathbf{\Psi}
\end{array}\right]|\mathbf{R}-d \mathbf{I}|^{-1}\left(\mathbf{Q}(\mathbf{R}-d \mathbf{I})^{-1}\right) \\
& =\left(\left[\begin{array}{ll}
\mathbf{I} & \mathbf{\Psi}
\end{array}\right]|\mathbf{R}-d \mathbf{I}|^{-1}\left(\mathbf{Q}(\mathbf{R}-d \mathbf{I})^{-1}\right)^{i-1}\right)\left(\mathbf{Q}(\mathbf{R}-d \mathbf{I})^{-1}\right),
\end{aligned}
$$

that equals to (48). Identity (49) can be proven similarly.

Theorem 2. For the following integral we have

$$
\int_{x=0}^{\infty} \mathbf{A}(t, x)\left[\begin{array}{c}
\mathbf{\Psi} \\
\mathbf{I}
\end{array}\right] e^{\mathbf{U} x} \mathrm{~d} x=\left[\begin{array}{c}
\mathbf{\Psi} \\
\mathbf{I}
\end{array}\right] e^{d \mathbf{U} t}
$$


Proof. Let us multiply both sides of (4) by $\left[\begin{array}{c}\mathbf{\Psi} \\ \mathbf{I}\end{array}\right] e^{\mathbf{U} x}$ and integrate from 0 to $\infty$. We get

$$
\frac{\partial}{\partial t} \int_{x=0}^{\infty} \mathbf{A}(t, x)\left[\begin{array}{c}
\mathbf{\Psi} \\
\mathbf{I}
\end{array}\right] e^{\mathbf{U} x} \mathrm{~d} x+\int_{x=0}^{\infty} \frac{\partial}{\partial x} \mathbf{A}(t, x) \mathbf{R}\left[\begin{array}{c}
\mathbf{\Psi} \\
\mathbf{I}
\end{array}\right] e^{\mathbf{U} x} \mathrm{~d} x=\int_{x=0}^{\infty} \mathbf{A}(t, x) \mathbf{Q}\left[\begin{array}{c}
\mathbf{\Psi} \\
\mathbf{I}
\end{array}\right] e^{\mathbf{U} x} \mathrm{~d} x
$$

The integration of the second term by parts leads to

$$
\frac{\partial}{\partial t} \int_{x=0}^{\infty} \mathbf{A}(t, x)\left[\begin{array}{c}
\mathbf{\Psi} \\
\mathbf{I}
\end{array}\right] e^{\mathbf{U} x} \mathrm{~d} x-\int_{x=0}^{\infty} \mathbf{A}(t, x) \mathbf{R}\left[\begin{array}{c}
\mathbf{\Psi} \\
\mathbf{I}
\end{array}\right] \mathbf{U} e^{\mathbf{U} x} \mathrm{~d} x=\int_{x=0}^{\infty} \mathbf{A}(t, x) \mathbf{Q}\left[\begin{array}{c}
\mathbf{\Psi} \\
\mathbf{I}
\end{array}\right] e^{\mathbf{U} x} \mathrm{~d} x .
$$

Applying Lemma 4 this expression simplifies to

$$
\frac{\partial}{\partial t}\left(\int_{x=0}^{\infty} \mathbf{A}(t, x)\left[\begin{array}{c}
\mathbf{\Psi} \\
\mathbf{I}
\end{array}\right] e^{\mathbf{U} x} \mathrm{~d} x\right)=\left(\int_{x=0}^{\infty} \mathbf{A}(t, x)\left[\begin{array}{c}
\mathbf{\Psi} \\
\mathbf{I}
\end{array}\right] e^{\mathbf{U} x} \mathrm{~d} x\right) d \mathbf{U}
$$

which establishes the theorem.

Corollary 2. From Theorem 2 and Lemma 3 we also have

$$
\begin{gathered}
\int_{x=0}^{\infty} \mathbf{A}(t, x)\left[\begin{array}{c}
\mathbf{\Psi} \\
\mathbf{I}
\end{array}\right](\mathbf{I}-\hat{\mathbf{\Psi}} \mathbf{\Psi})^{-1} e^{\hat{\mathbf{K}} x} \mathrm{~d} x=\left[\begin{array}{c}
\mathbf{\Psi} \\
\mathbf{I}
\end{array}\right](\mathbf{I}-\hat{\mathbf{\Psi}} \mathbf{\Psi})^{-1} e^{d \hat{\mathbf{K}} t}, \\
\int_{x=0}^{\infty} \mathbf{A}(t, x)\left[\begin{array}{c}
\mathbf{I} \\
\hat{\mathbf{\Psi}}
\end{array}\right](\mathbf{I}-\mathbf{\Psi} \hat{\mathbf{\Psi}})^{-1} e^{\mathbf{K} x} \mathrm{~d} x=\left[\begin{array}{c}
\mathbf{I} \\
\hat{\mathbf{\Psi}}
\end{array}\right](\mathbf{I}-\mathbf{\Psi} \hat{\mathbf{\Psi}})^{-1} e^{d \mathbf{K} t} .
\end{gathered}
$$

Theorem 3. The following matrix identity holds for $i \geq 1$

$$
(\mathbf{R}-d \mathbf{I})\left(\left[\begin{array}{c}
\mathbf{I} \\
\hat{\mathbf{\Psi}}
\end{array}\right](\mathbf{I}-\mathbf{\Psi} \hat{\mathbf{\Psi}})^{-1} \mathbf{K}^{i}\left[\begin{array}{ll}
\mathbf{I} & \mathbf{\Psi}
\end{array}\right]-\left[\begin{array}{c}
\mathbf{\Psi} \\
\mathbf{I}
\end{array}\right](\mathbf{I}-\hat{\mathbf{\Psi}} \mathbf{\Psi})^{-1}(-\hat{\mathbf{K}})^{i}\left[\begin{array}{ll}
\hat{\mathbf{\Psi}} & \mathbf{I}
\end{array}\right]\right)|\mathbf{R}-d \mathbf{I}|^{-1}=\left(\mathbf{Q}(\mathbf{R}-d \mathbf{I})^{-1}\right)^{i}
$$

Proof. For $i=0$ the identity can de checked in a direct way by exploiting $\hat{\mathbf{\Psi}}(\mathbf{I}-\mathbf{\Psi} \hat{\mathbf{\Psi}})^{-1}=(\mathbf{I}-\hat{\mathbf{\Psi}} \mathbf{\Psi})^{-1} \hat{\mathbf{\Psi}}$, $\mathbf{\Psi}(\mathbf{I}-\hat{\mathbf{\Psi}} \mathbf{\Psi})^{-1}=(\mathbf{I}-\mathbf{\Psi} \hat{\mathbf{\Psi}})^{-1} \mathbf{\Psi}$ and that $(\mathbf{I}-\mathbf{\Psi} \hat{\mathbf{\Psi}})^{-1} \mathbf{\Psi} \hat{\mathbf{\Psi}}=(\mathbf{I}-\mathbf{\Psi} \hat{\mathbf{\Psi}})^{-1}-\mathbf{I}$.

For $i>0$ the proof is based on induction. Assume that (57) holds for $i-1$. For $i$ the left hand side is transformed using Corollary 1 as

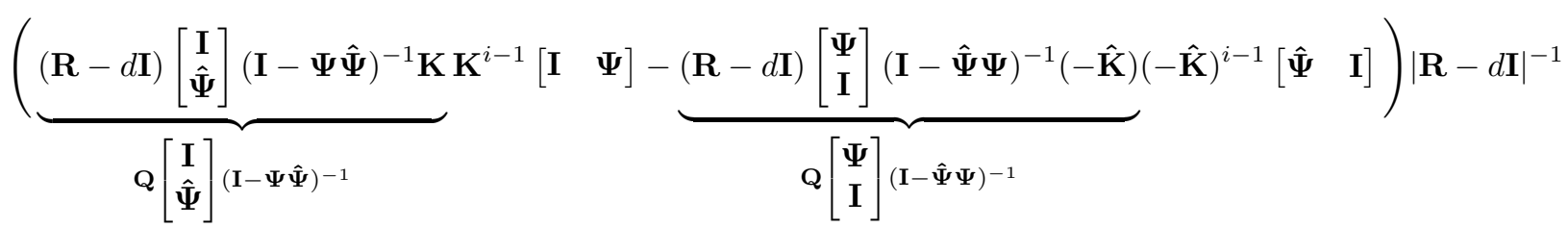

$$
\begin{aligned}
& =\mathbf{Q}(\mathbf{R}-d \mathbf{I})^{-1} \underbrace{(\mathbf{R}-d \mathbf{I})\left(\left[\begin{array}{c}
\mathbf{I} \\
\mathbf{\Psi}
\end{array}\right](\mathbf{I}-\mathbf{\Psi} \hat{\mathbf{\Psi}})^{-1} \mathbf{K}^{i-1}\left[\begin{array}{ll}
\mathbf{I} & \mathbf{\Psi}
\end{array}\right]-\left[\begin{array}{c}
\mathbf{\Psi} \\
\mathbf{I}
\end{array}\right](\mathbf{I}-\hat{\mathbf{\Psi}} \mathbf{\Psi})^{-1}(-\hat{\mathbf{K}})^{i-1}[\hat{\mathbf{\Psi}} \quad \mathbf{I}]\right)|\mathbf{R}-d \mathbf{I}|^{-1}} \\
& =\left(\mathbf{Q}(\mathbf{R}-d \mathbf{I})^{-1}\right)^{i} . \\
& \left(\mathbf{Q}(\mathbf{R}-d \mathbf{I})^{-1}\right)^{i-1}
\end{aligned}
$$

\subsection{The phase probability vector at the beginning of the vacation}

Theorem 4. The initial vector $\beta$ is given by $\beta=\left[\begin{array}{ll}0 & \omega\end{array}\right]$, where size $N^{-}$vector $\omega$ is the solution to the linear system $\omega \mathbf{U}=0, \omega \mathbb{1}=1$. 
ProOf. At the end of the busy period the background process must be in $\mathcal{S}^{-}$, hence the entries of $\beta=$ $\left[\begin{array}{ll}\beta^{+} & \beta^{-}\end{array}\right]$belonging to positive states, are zeroes (hence, $\beta^{+}=0$ ).

According to [17, Theorem 2] the phase transitions of a fluid model over the busy period are given by $\left[\begin{array}{c}\Psi \\ \mathbf{I}\end{array}\right] e^{\mathbf{U} x}$, assuming that the initial fluid level is $x$. Thus, by conditioning on the amount of fluid accumulated during the vacation we get the following equation for $\beta$,

$$
\left[\begin{array}{ll}
\beta^{+} & \beta^{-}
\end{array}\right] \int_{x=0}^{\infty} \mathbf{A}(t, x)\left[\begin{array}{c}
\mathbf{\Psi} \\
\mathbf{I}
\end{array}\right] e^{\mathbf{U} x} \mathrm{~d} x=\beta^{-} .
$$

Applying Theorem 2 simplifies this equation to

$$
\left[\beta^{+} \beta^{-}\right]\left[\begin{array}{c}
\mathbf{\Psi} \\
\mathbf{I}
\end{array}\right] e^{d \mathbf{U}^{t}}=\beta^{-}
$$

which is clearly satisfied by $\beta=\left[\begin{array}{ll}0 & \omega\end{array}\right]$.

Theorem 5. The initial probability vector $\beta$ and the stationary probability vector of the background CTMC $\pi$ are related by

$$
\beta=\frac{1}{\lambda-d} \pi(\mathbf{R}-d \mathbf{I})\left[\begin{array}{cc}
\mathbf{I} & \mathbf{\Psi} \\
\hat{\mathbf{\Psi}} & \mathbf{I}
\end{array}\right]
$$

Proof. From (39) we have that $\pi(\mathbf{R}-d \mathbf{I})\left[\begin{array}{c}\mathbf{\Psi} \\ \mathbf{I}\end{array}\right] \mathbf{U}=0$ and from (40) we have that $\pi(\mathbf{R}-d \mathbf{I})\left[\begin{array}{c}\mathbf{I} \\ \hat{\mathbf{\Psi}}\end{array}\right]=0$, providing the theorem. The term $1 /(\lambda-d)$ is the normalization constant.

\subsection{Fluid density level during the vacation period}

The LT of $L^{(v)}(y)$, which is the first term of (37), can be expressed as

$$
\begin{aligned}
L^{(v) *}(s) & =\int_{y=0}^{\infty} e^{-s y} L^{(v)}(y) \mathrm{d} y=\int_{u=0}^{\infty} \sigma(u) \beta \int_{x=0}^{u} \int_{y=0}^{\infty} e^{-s y} \mathbf{A}(x, y) \mathrm{d} y \mathrm{~d} x \mathrm{~d} u \\
& =\int_{u=0}^{\infty} \sigma(u) \beta \int_{x=0}^{u} e^{(\mathbf{Q}-s \mathbf{R}) x} \mathrm{~d} x \mathrm{~d} u=\int_{u=0}^{\infty} \sigma(u) \beta\left(\mathbf{I}-e^{(\mathbf{Q}-s \mathbf{R}) u}\right)(-\mathbf{Q}+s \mathbf{R})^{-1} \mathrm{~d} u \\
& =\beta\left(\mathbf{I}-\sigma^{*}(s \mathbf{R}-\mathbf{Q})\right)(-\mathbf{Q}+s \mathbf{R})^{-1} .
\end{aligned}
$$

We recall that $\sigma^{*}(\mathbf{X})$ with square matrix $\mathbf{X}$ is defined by $\int_{u=0}^{\infty} \sigma(u) e^{-\mathbf{X} u} \mathrm{~d} u$.

4.4. The expected number of level crossings during the service period

$L^{(s)}(y)$ is obtained by substituting $(32)$ and (33) into the definition (37). For the LT $L^{(s) *}(s)=$ $\int_{y=0}^{\infty} e^{-s y} L^{(s)}(y) \mathrm{d} y$ we get

$$
\begin{aligned}
& L^{(s) *}(s)=\int_{u=0}^{\infty} \sigma(u) \int_{y=0}^{\infty} e^{-s y} \int_{x=0}^{\infty} \beta \mathbf{A}(u, x) \mathbf{M}(x, y) \mathrm{d} x \mathrm{~d} y \mathrm{~d} u
\end{aligned}
$$

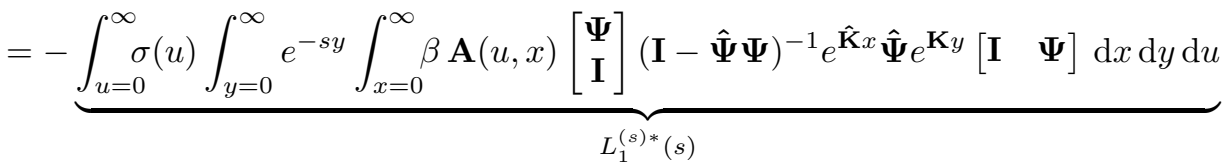

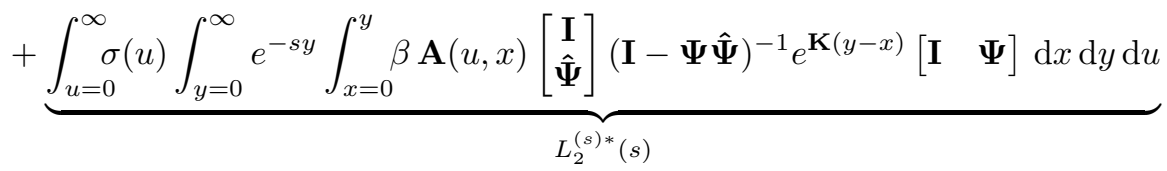

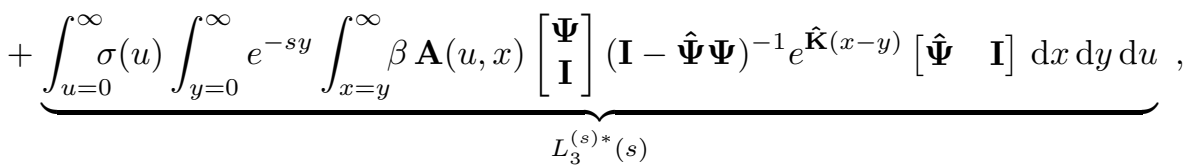


which consists of three terms.

Making use of Corollary 2 and Lemma 3 the first term is expressed by

$$
\begin{aligned}
L_{1}^{(s) *}(s) & =\int_{u=0}^{\infty} \sigma(u) \int_{x=0}^{\infty} \beta \mathbf{A}(u, x)\left[\begin{array}{c}
\mathbf{\Psi} \\
\mathbf{I}
\end{array}\right](\mathbf{I}-\hat{\mathbf{\Psi}} \mathbf{\Psi})^{-1} e^{\hat{\mathbf{K}} x} \hat{\mathbf{\Psi}}(s \mathbf{I}-\mathbf{K})^{-1}\left[\begin{array}{ll}
\mathbf{I} & \mathbf{\Psi}
\end{array}\right] \mathrm{d} x \mathrm{~d} u \\
& =\int_{u=0}^{\infty} \sigma(u)\left[\begin{array}{ll}
0 & \omega
\end{array}\right]\left[\begin{array}{c}
\mathbf{\Psi} \\
\mathbf{I}
\end{array}\right](\mathbf{I}-\hat{\mathbf{\Psi}} \mathbf{\Psi})^{-1} e^{d \hat{\mathbf{K}} u} \hat{\mathbf{\Psi}}(s \mathbf{I}-\mathbf{K})^{-1}\left[\begin{array}{ll}
\mathbf{I} & \mathbf{\Psi}
\end{array}\right] \mathrm{d} u \\
& =\int_{u=0}^{\infty} \sigma(u) \omega e^{d \mathbf{U} u}(\mathbf{I}-\hat{\mathbf{\Psi}} \mathbf{\Psi})^{-1} \hat{\mathbf{\Psi}}(s \mathbf{I}-\mathbf{K})^{-1}\left[\begin{array}{ll}
\mathbf{I} & \mathbf{\Psi}
\end{array}\right] \mathrm{d} u \\
& =\omega(\mathbf{I}-\hat{\mathbf{\Psi}} \mathbf{\Psi})^{-1} \hat{\mathbf{\Psi}}(s \mathbf{I}-\mathbf{K})^{-1}\left[\begin{array}{ll}
\mathbf{I} & \mathbf{\Psi}
\end{array}\right] \\
& =\omega \hat{\mathbf{\Psi}}(\mathbf{I}-\mathbf{\Psi} \hat{\mathbf{\Psi}})^{-1}(s \mathbf{I}-\mathbf{K})^{-1}\left[\begin{array}{ll}
\mathbf{I} & \mathbf{\Psi}
\end{array}\right] .
\end{aligned}
$$

For the second term we have

$$
\begin{aligned}
L_{2}^{(s) *}(s) & =\int_{u=0}^{\infty} \sigma(u) \int_{x=0}^{\infty} \int_{y=x}^{\infty} e^{-s x} e^{-s(y-x)} \beta \mathbf{A}(u, x)\left[\begin{array}{c}
\mathbf{I} \\
\hat{\mathbf{\Psi}}
\end{array}\right](\mathbf{I}-\mathbf{\Psi} \hat{\mathbf{\Psi}})^{-1} e^{\mathbf{K}(y-x)}\left[\begin{array}{ll}
\mathbf{I} & \mathbf{\Psi}
\end{array}\right] \mathrm{d} y \mathrm{~d} x \mathrm{~d} u \\
& =\int_{u=0}^{\infty} \sigma(u) \beta \int_{x=0}^{\infty} e^{-s x} \mathbf{A}(u, x)\left[\begin{array}{c}
\mathbf{I} \\
\hat{\mathbf{\Psi}}
\end{array}\right](\mathbf{I}-\mathbf{\Psi} \hat{\mathbf{\Psi}})^{-1} \int_{y=x}^{\infty} e^{-s(y-x)} e^{\mathbf{K}(y-x)}\left[\begin{array}{ll}
\mathbf{I} & \mathbf{\Psi}
\end{array}\right] \mathrm{d} y \mathrm{~d} x \mathrm{~d} u \\
& =\beta \sigma^{*}(s \mathbf{R}-\mathbf{Q})\left[\begin{array}{c}
\mathbf{I} \\
\hat{\mathbf{\Psi}}
\end{array}\right](\mathbf{I}-\mathbf{\Psi} \hat{\mathbf{\Psi}})^{-1}(s \mathbf{I}-\mathbf{K})^{-1}\left[\begin{array}{ll}
\mathbf{I} & \mathbf{\Psi}
\end{array}\right] .
\end{aligned}
$$

The third term is transformed by swapping the sums and applying Corollary 2 and Lemma 3 again, yielding

$$
\begin{aligned}
& L_{3}^{(s) *}(s)=\int_{u=0}^{\infty} \sigma(u) \int_{x=0}^{\infty} \int_{y=0}^{x} e^{-s x} e^{s(x-y)} \beta \mathbf{A}(u, x)\left[\begin{array}{c}
\mathbf{\Psi} \\
\mathbf{I}
\end{array}\right](\mathbf{I}-\hat{\mathbf{\Psi} \Psi})^{-1} e^{\hat{\mathbf{K}}(x-y)}\left[\begin{array}{ll}
\hat{\mathbf{\Psi}} & \mathbf{I}
\end{array}\right] \mathrm{d} y \mathrm{~d} x \mathrm{~d} u \\
& =\int_{u=0}^{\infty} \sigma(u) \beta \int_{x=0}^{\infty} e^{-s x} \mathbf{A}(u, x)\left[\begin{array}{c}
\mathbf{\Psi} \\
\mathbf{I}
\end{array}\right](\mathbf{I}-\hat{\mathbf{\Psi}} \mathbf{\Psi})^{-1} \int_{y=0}^{x} e^{s(x-y)} e^{\hat{\mathbf{K}}(x-y)}\left[\begin{array}{ll}
\hat{\mathbf{\Psi}} & \mathbf{I}
\end{array}\right] \mathrm{d} y \mathrm{~d} x \mathrm{~d} u \\
& =\int_{u=0}^{\infty} \sigma(u) \beta \int_{x=0}^{\infty} e^{-s x} \mathbf{A}(u, x)\left[\begin{array}{c}
\mathbf{\Psi} \\
\mathbf{I}
\end{array}\right](\mathbf{I}-\hat{\mathbf{\Psi}} \mathbf{\Psi})^{-1}\left(\mathbf{I}-e^{(s \mathbf{I}+\hat{\mathbf{K}}) x}\right)(-s \mathbf{I}-\hat{\mathbf{K}})^{-1}\left[\begin{array}{ll}
\hat{\Psi} & \mathbf{I}
\end{array}\right] \mathrm{d} x \mathrm{~d} u \\
& =\beta \sigma^{*}(s \mathbf{R}-\mathbf{Q})\left[\begin{array}{c}
\mathbf{\Psi} \\
\mathbf{I}
\end{array}\right](\mathbf{I}-\hat{\mathbf{\Psi}} \mathbf{\Psi})^{-1}(-s \mathbf{I}-\hat{\mathbf{K}})^{-1}\left[\begin{array}{ll}
\hat{\mathbf{\Psi}} & \mathbf{I}
\end{array}\right]-\omega(\mathbf{I}-\hat{\mathbf{\Psi}} \mathbf{\Psi})^{-1}(-s \mathbf{I}-\hat{\mathbf{K}})^{-1}\left[\begin{array}{ll}
\hat{\mathbf{\Psi}} & \mathbf{I}
\end{array}\right] .
\end{aligned}
$$

\subsection{The stationary distribution of the fluid level}

We conclude the results collected in the previous subsections in the following theorem

Theorem 6. The vector Laplace transform of the stationary distribution of the fluid level is given by

$$
q^{*}(s)=\frac{s d}{c} \beta\left(\sigma^{*}(s \mathbf{R}-\mathbf{Q})-\mathbf{I}\right)(s \mathbf{R}-\mathbf{Q})^{-1}(s(\mathbf{R}-d \mathbf{I})-\mathbf{Q})^{-1} .
$$

Proof. Substituting $L^{(v) *}(s), L_{1}^{(s) *}(s), L_{2}^{(s) *}(s)$ and $L_{3}^{(s) *}(s)$ into the transform version of (37)

$$
q^{*}(s)=L^{(v) *}(s)+\left(-L_{1}^{(s) *}(s)+L_{2}^{(s) *}(s)+L_{3}^{(s) *}(s)\right) \cdot|\mathbf{R}-d \mathbf{I}|^{-1}
$$

and exploiting that $\omega \hat{\mathbf{\Psi}}=\beta\left[\begin{array}{c}\mathbf{I} \\ \hat{\mathbf{\Psi}}\end{array}\right]$ and that $\omega=\beta\left[\begin{array}{c}\mathbf{\Psi} \\ \mathbf{I}\end{array}\right]$ we obtain

$$
\begin{aligned}
q^{*}(s)=\frac{1}{c} \beta & \left(\sigma^{*}(s \mathbf{R}-\mathbf{Q})-\mathbf{I}\right) \\
& \times\left(\left[\begin{array}{c}
\mathbf{\Psi} \\
\mathbf{I}
\end{array}\right](\mathbf{I}-\hat{\mathbf{\Psi}} \mathbf{\Psi})^{-1}(-s \mathbf{I}-\hat{\mathbf{K}})^{-1}\left[\begin{array}{ll}
\hat{\mathbf{\Psi}} & \mathbf{I}
\end{array}\right]|\mathbf{R}-d \mathbf{I}|^{-1}\right. \\
& \left.+\left[\begin{array}{c}
\mathbf{I} \\
\hat{\mathbf{\Psi}}
\end{array}\right](\mathbf{I}-\mathbf{\Psi} \hat{\mathbf{\Psi}})^{-1}(s \mathbf{I}-\mathbf{K})^{-1}\left[\begin{array}{ll}
\mathbf{I} & \mathbf{\Psi}
\end{array}\right]|\mathbf{R}-d \mathbf{I}|^{-1}+(\mathbf{Q}-s \mathbf{R})^{-1}\right),
\end{aligned}
$$


which can be expressed due to Theorem 3 as

$$
\begin{aligned}
q^{*}(s) & =\frac{1}{c} \beta\left(\sigma^{*}(s \mathbf{R}-\mathbf{Q})-\mathbf{I}\right)\left((\mathbf{R}-d \mathbf{I})^{-1}\left(s \mathbf{I}-\mathbf{Q}(\mathbf{R}-d \mathbf{I})^{-1}\right)^{-1}+(\mathbf{Q}-s \mathbf{R})^{-1}\right) \\
& =\frac{1}{c} \beta\left(\sigma^{*}(s \mathbf{R}-\mathbf{Q})-\mathbf{I}\right)\left((s(\mathbf{R}-d \mathbf{I})-\mathbf{Q})^{-1}+(\mathbf{Q}-s \mathbf{R})^{-1}\right) \\
& =\frac{1}{c} \beta\left(\sigma^{*}(s \mathbf{R}-\mathbf{Q})-\mathbf{I}\right)(\mathbf{Q}-s \mathbf{R})^{-1}\left((\mathbf{Q}+s d \mathbf{I}-s d \mathbf{I}-s \mathbf{R})(s(\mathbf{R}-d \mathbf{I})-\mathbf{Q})^{-1}+\mathbf{I}\right) \\
& =\frac{1}{c} \beta\left(\sigma^{*}(s \mathbf{R}-\mathbf{Q})-\mathbf{I}\right)(\mathbf{Q}-s \mathbf{R})^{-1}\left(-s d(s(\mathbf{R}-d \mathbf{I})-\mathbf{Q})^{-1}\right) \\
& =\frac{s d}{c} \beta\left(\sigma^{*}(s \mathbf{R}-\mathbf{Q})-\mathbf{I}\right)(s \mathbf{R}-\mathbf{Q})^{-1}(s(\mathbf{R}-d \mathbf{I})-\mathbf{Q})^{-1}
\end{aligned}
$$

\subsection{Relation to previous results}

Following the methodology of discrete vacation and polling models the fluid vacation models with gated discipline [12] and with exhaustive discipline without positive rate during service [13] have been analyzed based on the Laplace transform description of the fluid level distribution at the beginning and the end of the service period, $\mathbf{f}^{*}(s)$ and $\mathbf{m}^{*}(s)$, respectively, with elements $\mathbf{f}^{*}(s)_{\ell}=\lim _{i \rightarrow \infty} E\left(e^{-s \mathcal{X}\left(t_{i}^{(s)}\right) \mathcal{I}\left\{\Omega\left(t_{i}^{(s)}\right)=\ell\right\}}\right)$ and $\mathbf{m}^{*}(s)_{\ell}=\lim _{i \rightarrow \infty} E\left(e^{-s \mathcal{X}\left(t_{i}^{(v)}\right)} \mathcal{I}\left\{\Omega\left(t_{i}^{(v)}\right)=\ell\right\}\right)$, where $t_{i}^{(s)}\left(t_{i}^{(v)}\right)$ denotes the $i$ th service (vacation) instance.

In case of fluid vacation models with exhaustive discipline the fluid level is zero at vacation start epoch and only the distribution of the background Markov chain plays role. For possible positive fluid rate during service the distribution is provided by Theorem 4 , that is

$$
\mathbf{m}^{*}(s)=\beta=\left[\begin{array}{ll}
0 & \omega
\end{array}\right] .
$$

Starting the $\sigma$ long vacation period with distribution $\mathbf{m}^{*}(s)$ the fluid distribution at the end of the vacation period is $[12,13]$

$$
\mathbf{f}^{*}(s)=\mathbf{m}^{*}(s) \sigma^{*}(s \mathbf{R}-\mathbf{Q})=\beta \sigma^{*}(s \mathbf{R}-\mathbf{Q}),
$$

from which

$$
\mathbf{f}^{*}(s)-\mathbf{m}^{*}(s)=\beta\left(\sigma^{*}(s \mathbf{R}-\mathbf{Q})-\mathbf{I}\right) .
$$

Substituting this expressions into (62), multiplying both sides with $(s \mathbf{R}-\mathbf{Q})$ and $(s(\mathbf{R}-d \mathbf{I})-\mathbf{Q})$ and using the commutativity of these two matrices we obtain

$$
q^{*}(s)(s \mathbf{R}-\mathbf{Q})(s(\mathbf{R}-d \mathbf{I})-\mathbf{Q})=\frac{s d}{c}\left(\mathbf{f}^{*}(s)-\mathbf{m}^{*}(s)\right),
$$

which is identical with $[12$, eq. (58)] and [13, eq. (33)]. It means that similar to the case of discrete vacation models there are general discipline independent transform domain expressions for fluid vacation models as well and (64) is one of them. The validity of (64) for exhaustive fluid vacation models with positive rate during service was a huge surprise for us. Results like Theorem 1 suggests no hope for such simple relation. Theorem 3 is the key result which relates the complex level crossing expressions with simple $\mathbf{Q}$ and $\mathbf{R}$ related ones. Substituting (63) and (60) into (64) we can write

$$
q^{*}(s)(s \mathbf{R}-\mathbf{Q})(s(\mathbf{R}-d \mathbf{I})-\mathbf{Q})=\frac{s}{E(\sigma)} \pi(\mathbf{R}-d \mathbf{I})\left[\begin{array}{cc}
\mathbf{I} & \mathbf{\Psi} \\
\hat{\mathbf{\Psi}} & \mathbf{I}
\end{array}\right] \sigma^{*}(s \mathbf{R}-\mathbf{Q}) .
$$

In this expression we have a series of directly computable $\mathbf{Q}$ and $\mathbf{R}$ related expressions, e.g., $\pi$ and $\sigma^{*}(s \mathbf{R}-\mathbf{Q})$, and the effect of the involved fluid model analysis is concentrated in a single matrix with $\boldsymbol{\Psi}$ and $\hat{\boldsymbol{\Psi}}$. 


\section{Mean fluid level}

The derivatives of $q^{*}(s)$ at $s \rightarrow 0$ provide the moments of the fluid level, $q^{(i)}=\left.(-1)^{i} \frac{\mathrm{d}^{i}}{\mathrm{~d} s^{i}} q^{*}(s)\right|_{s \rightarrow 0}$.

Theorem 7. The state dependent stationary mean fluid level is given by

$$
q^{(1)}=q^{(1)} \mathbb{1} \pi+\left(\pi \mathbf{R} \mathbf{Q}+\frac{1}{E(\sigma)} \pi(\mathbf{R}-d \mathbf{I})\left[\begin{array}{cc}
\mathbf{I} & \mathbf{\Psi} \\
\hat{\mathbf{\Psi}} & \mathbf{I}
\end{array}\right] \sigma^{*}(-\mathbf{Q})\right)\left(\mathbb{1} \pi-\mathbf{Q}^{2}\right)^{-1} .
$$

Proof. Considering that $q^{*}(0)=q^{(0)}=\pi$ the derivative of $(65)$ at $s=0$ gives

$$
-q^{(1)} \mathbf{Q}^{2}-\pi \mathbf{R} \mathbf{Q}-\underbrace{\pi \mathbf{Q}}_{0}(\mathbf{R}-d \mathbf{I})=\frac{1}{E(\sigma)} \pi(\mathbf{R}-d \mathbf{I})\left[\begin{array}{cc}
\mathbf{I} & \mathbf{\Psi} \\
\hat{\mathbf{\Psi}} & \mathbf{I}
\end{array}\right] \sigma^{*}(-\mathbf{Q}) .
$$

Adding $q^{(1)} \mathbb{1} \pi$ to both sides, using that $\left(\mathbb{1} \pi-\mathbf{Q}^{2}\right)$ is non-singular and $\pi\left(\mathbb{1} \pi-\mathbf{Q}^{2}\right)^{-1}=\pi$ we obtain (66).

The only remaining unknown of (66) is the overall mean fluid level, the scalar $q^{(1)} \mathbb{1}$, unfortunately the analysis of $q^{(1)} \mathbb{1}$ is not straightforward and the rest of the section is devoted to this problem.

Lemma 6. Let square matrix $\mathbf{X}$ have a single zero eigenvalue and let the associated normalized left and right eigenvectors be $x_{l}$ and $x_{r}^{T}$, respectively, that is $x_{l} \neq 0, x_{r}^{T} \neq 0, x_{l} \mathbf{X}=0, \mathbf{X} x_{r}^{T}=0, x_{l} x_{r}^{T}=1$. For $\mathbf{X}$ we have

$$
\begin{aligned}
\lim _{s \rightarrow 0} s(s \mathbf{I}-\mathbf{X})^{-1} & =\lim _{s \rightarrow 0} \mathbf{I}+\mathbf{X}(s \mathbf{I}-\mathbf{X})^{-1}=x_{r}^{T} x_{l}, \\
\lim _{s \rightarrow 0} \frac{\mathrm{d}}{\mathrm{d} s} s(s \mathbf{I}-\mathbf{X})^{-1} & =\lim _{s \rightarrow 0} \frac{\mathrm{d}}{\mathrm{d} s} \mathbf{X}(s \mathbf{I}-\mathbf{X})^{-1}=-\mathbf{X}\left(x_{r}^{T} x_{l}-\mathbf{X}\right)^{-2}=\left(x_{r}^{T} x_{l}-\mathbf{X}\right)^{-1}-x_{r}^{T} x_{l} .
\end{aligned}
$$

Proof. First we note that $s(s \mathbf{I}-\mathbf{X})^{-1}=(s \mathbf{I}-\mathbf{X}+\mathbf{X})(s \mathbf{I}-\mathbf{X})^{-1}=\mathbf{I}+\mathbf{X}(s \mathbf{I}-\mathbf{X})^{-1},\left(\mathbf{X}-x_{r}^{T} x_{l}\right)$ is a non-singular matrix, since the 0 eigenvalue of $\mathbf{X}$ is moved to -1 by subtracting $x_{r}^{T} x_{l}$ and all other eigenvalues are untouched, and by the matrix inversion lemma we have

$$
(\mathbf{A}+u v)^{-1}=\mathbf{A}^{-1}-\frac{\mathbf{A}^{-1} u v \mathbf{A}^{-1}}{1+v \mathbf{A}^{-1} u},
$$

where $\mathbf{A}$ is a non-singular matrix $u$ is a column vector and $v$ is a row vector. Applying the matrix inversion lemma with $\mathbf{A}=s \mathbf{I}-\mathbf{X}+x_{r}^{T} x_{l}, u=x_{r}^{T}, v=x_{l}$ we obtain

$$
\begin{aligned}
(s \mathbf{I}-\mathbf{X})^{-1} & =\left(s \mathbf{I}-\mathbf{X}+x_{r}^{T} x_{l}-x_{r}^{T} x_{l}\right)^{-1}=\left(s \mathbf{I}-\mathbf{X}+x_{r}^{T} x_{l}\right)^{-1}+\frac{\left(s \mathbf{I}-\mathbf{X}+x_{r}^{T} x_{l}\right)^{-1} x_{r}^{T} x_{l}\left(s \mathbf{I}-\mathbf{X}+x_{r}^{T} x_{l}\right)^{-1}}{1-x_{l}\left(s \mathbf{I}-\mathbf{X}+x_{r}^{T} x_{l}\right)^{-1} x_{r}^{T}} \\
& =\left(s \mathbf{I}-\mathbf{X}+x_{r}^{T} x_{l}\right)^{-1}+\frac{\frac{1}{s+1} x_{r}^{T} x_{l} \frac{1}{s+1}}{1-x_{l} \frac{1}{s+1} x_{r}^{T}}=\left(s \mathbf{I}-\mathbf{X}+x_{r}^{T} x_{l}\right)^{-1}+\frac{1}{s+1} \frac{x_{r}^{T} x_{l}}{s+1-x_{l} x_{r}^{T}} \\
& =\left(s \mathbf{I}-\mathbf{X}+x_{r}^{T} x_{l}\right)^{-1}+\frac{1}{s(s+1)} x_{r}^{T} x_{l},
\end{aligned}
$$

where we used

$$
\left(s \mathbf{I}-\mathbf{X}+x_{r}^{T} x_{l}\right)^{-1} x_{r}^{T}=\frac{1}{s+1} x_{r}^{T} \quad \text { and } \quad x_{l}\left(s \mathbf{I}-\mathbf{X}+x_{r}^{T} x_{l}\right)^{-1}=x_{l} \frac{1}{s+1},
$$

which comes from

$$
\left(s \mathbf{I}-\mathbf{X}+x_{r}^{T} x_{l}\right) x_{r}^{T}=(s+1) x_{r}^{T} \quad \text { and } \quad x_{l}\left(s \mathbf{I}-\mathbf{X}+x_{r}^{T} x_{l}\right)=(s+1) x_{l} .
$$


Using (69) for the first limit we have

$$
\lim _{s \rightarrow 0} s(s \mathbf{I}-\mathbf{X})^{-1}=\lim _{s \rightarrow 0} s(s \mathbf{I} \underbrace{-\mathbf{X}+x_{r}^{T} x_{l}}_{\text {non-singular }})^{-1}+\frac{s}{s(s+1)} x_{r}^{T} x_{l}=x_{r}^{T} x_{l} .
$$

Since the derivatives of $(s \mathbf{I}-\mathbf{X})$ with respect to $s$ commute, we can obtain the derivatives of $(s \mathbf{I}-\mathbf{X})^{-1}$ according to the scalar derivation rules

$$
\frac{\mathrm{d}}{\mathrm{d} s} s(s \mathbf{I}-\mathbf{X})^{-1}=(s \mathbf{I}-\mathbf{X})^{-1}-s(s \mathbf{I}-\mathbf{X})^{-2}=(s \mathbf{I}-\mathbf{X})^{-1}\left(\mathbf{I}-s(s \mathbf{I}-\mathbf{X})^{-1}\right) .
$$

Applying (69) again, for the second limit we have

$$
\begin{aligned}
& \lim _{s \rightarrow 0} \frac{\mathrm{d}}{\mathrm{d} s} s(s \mathbf{I}-\mathbf{X})^{-1}=\lim _{s \rightarrow 0}\left(\left(s \mathbf{I}-\mathbf{X}+x_{r}^{T} x_{l}\right)^{-1}+\frac{1}{s(s+1)} x_{r}^{T} x_{l}\right)\left(\mathbf{I}-s\left(s \mathbf{I}-\mathbf{X}+x_{r}^{T} x_{l}\right)^{-1}-\frac{s}{s(s+1)} x_{r}^{T} x_{l}\right) \\
& =\lim _{s \rightarrow 0}\left(s \mathbf{I}-\mathbf{X}+x_{r}^{T} x_{l}\right)^{-1}\left(\mathbf{I}-s\left(s \mathbf{I}-\mathbf{X}+x_{r}^{T} x_{l}\right)^{-1}\right)-\frac{s}{s(s+1)}\left(s \mathbf{I}-\mathbf{X}+x_{r}^{T} x_{l}\right)^{-1} x_{r}^{T} x_{l} \\
& \quad+\frac{1}{s(s+1)} x_{r}^{T} x_{l}\left(\mathbf{I}-s\left(s \mathbf{I}-\mathbf{X}+x_{r}^{T} x_{l}\right)^{-1}\right)-\frac{s}{s^{2}(s+1)^{2}} x_{r}^{T} x_{l} x_{r}^{T} x_{l} \\
& =\lim _{s \rightarrow 0}\left(s \mathbf{I}-\mathbf{X}+x_{r}^{T} x_{l}\right)^{-1}\left(\mathbf{I}-s\left(s \mathbf{I}-\mathbf{X}+x_{r}^{T} x_{l}\right)^{-1}\right)-\frac{s}{s(s+1)^{2}} x_{r}^{T} x_{l} \\
& \quad+\frac{1}{s(s+1)} x_{r}^{T} x_{l}-\frac{s}{s(s+1)^{2}} x_{r}^{T} x_{l}-\frac{s}{s^{2}(s+1)^{2}} x_{r}^{T} x_{l} \\
& =\lim _{s \rightarrow 0}\left(s \mathbf{I}-\mathbf{X}+x_{r}^{T} x_{l}\right)^{-1}\left(\mathbf{I}-s\left(s \mathbf{I}-\mathbf{X}+x_{r}^{T} x_{l}\right)^{-1}\right)-\frac{s}{s(s+1)^{2}} x_{r}^{T} x_{l} \\
& \quad+\frac{1}{s(s+1)} x_{r}^{T} x_{l}-\frac{s}{s(s+1)^{2}} x_{r}^{T} x_{l}-\frac{s}{s^{2}(s+1)^{2}} x_{r}^{T} x_{l}=\left(x_{r}^{T} x_{l}-\mathbf{X}\right)^{-1}-x_{r}^{T} x_{l} .
\end{aligned}
$$

Theorem 8. The mean fluid level of the fluid vacation queue is

$$
q^{(1)} \mathbb{1}=\lambda E(\sigma) \frac{1+c_{\sigma}^{2}}{2}+E(\tilde{q}),
$$

where $E(\tilde{q})$ is

$$
E(\tilde{q})=1+(d-\lambda) \beta(\mathbf{R}-d \mathbf{I})^{-1}\left(\frac{(\mathbf{R}-d \mathbf{I}) \mathbb{1}}{\lambda-d} \pi-\mathbf{Q}(\mathbf{R}-d \mathbf{I})^{-1}\right)^{-1} \mathbb{1} .
$$

Proof. To make the proof simpler it is beneficial to express $q^{*}(s)$ as the product of row vector $q_{1}^{*}(s)$ and matrix $q_{2}^{*}(s)$, where

$$
\begin{aligned}
& q_{1}^{*}(s)=\frac{d}{c} \beta\left(\sigma^{*}(s \mathbf{R}-\mathbf{Q})-\mathbf{I}\right)(s \mathbf{R}-\mathbf{Q})^{-1}, \\
& q_{2}^{*}(s)=s(s(\mathbf{R}-d \mathbf{I})-\mathbf{Q})^{-1} .
\end{aligned}
$$

First $\lim _{s \rightarrow 0} q_{1}^{*}(s)$ is derived, yielding

$$
\begin{aligned}
\lim _{s \rightarrow 0} q_{1}^{*}(s) & =-\lim _{s \rightarrow 0} \frac{d}{c} \beta\left(\int_{x=0}^{\infty} \sigma(x) \sum_{k=1}^{\infty} \frac{x^{k}(\mathbf{Q}-s \mathbf{R})^{k-1}}{k !} \mathrm{d} x\right) \\
& =-\frac{d}{c} \beta\left(\int_{x=0}^{\infty} \sigma(x) \sum_{k=1}^{\infty} \frac{x^{k} \lim _{s \rightarrow 0}(\mathbf{Q}-s \mathbf{R})^{k-1}}{k !}(\mathbf{Q}-\mathbb{1} \pi)(\mathbf{Q}-\mathbb{1} \pi)^{-1} \mathrm{~d} x\right) \\
& =-\frac{d}{c} \beta\left(\int_{x=0}^{\infty} \sigma(x)\left(e^{\mathbf{Q} x}-\mathbf{I}-x \mathbb{1} \pi\right) \mathrm{d} x(\mathbf{Q}-\mathbb{1} \pi)^{-1}\right) \\
& =-\frac{d}{c} \beta\left(\sigma^{*}(\mathbf{Q})-\mathbf{I}\right)(\mathbf{Q}-\mathbb{1} \pi)^{-1}-E(\sigma) \frac{d}{c} \pi .
\end{aligned}
$$


The $\operatorname{limit}_{\lim _{s \rightarrow 0}} q_{2}^{*}(s)$ is obtained by using Lemma 6 as

$$
\lim _{s \rightarrow 0} q_{2}^{*}(s)=\lim _{s \rightarrow 0} s(\mathbf{R}-d \mathbf{I})^{-1}\left(s \mathbf{I}-\mathbf{Q}(\mathbf{R}-d \mathbf{I})^{-1}\right)^{-1}=(\mathbf{R}-d \mathbf{I})^{-1} \frac{(\mathbf{R}-d \mathbf{I}) \mathbb{1}}{\lambda-d} \pi=\frac{1}{\lambda-d} \mathbb{1} \pi,
$$

since the left and right eigenvalues corresponding to the zero eigenvalue of $\mathbf{X}=\mathbf{Q}(\mathbf{R}-d \mathbf{I})^{-1}$ are $x_{l}=\pi$ and $x_{r}^{T}=(\mathbf{R}-d \mathbf{I}) \mathbb{1} /(\lambda-d)$, respectively.

The mean queue length is computed by taking the derivative of $q^{*}(s)$ at $s \rightarrow 0$. Hence,

$$
q^{(1)} \mathbb{1}=-\lim _{s \rightarrow 0} \frac{\mathrm{d}}{\mathrm{d} s} q^{*}(s) \mathbb{1}=-\lim _{s \rightarrow 0}\left(\frac{\mathrm{d}}{\mathrm{d} s} q_{1}^{*}(s)\right) q_{2}^{*}(s) \mathbb{1}-\lim _{s \rightarrow 0} q_{1}^{*}(s)\left(\frac{\mathrm{d}}{\mathrm{d} s} q_{2}^{*}(s) \mathbb{1}\right),
$$

which consists of two terms. Let us start with the first term.

$$
\begin{aligned}
\lim _{s \rightarrow 0}\left(\frac{\mathrm{d}}{\mathrm{d} s} q_{1}^{*}(s)\right) q_{2}^{*}(s) \mathbb{1} & =-\lim _{s \rightarrow 0} \int_{x=0}^{\infty} \sigma(x) \beta \frac{\mathrm{d}}{\mathrm{d} s} \sum_{k=1}^{\infty} \frac{x^{k}}{k !}(\mathbf{Q}-s \mathbf{R})^{k-1} \mathrm{~d} x \mathbb{1} \pi \frac{d}{\lambda-d} \frac{1}{c} \mathbb{1} \\
& =\int_{x=0}^{\infty} \sigma(x) \beta \sum_{k=2}^{\infty} \frac{x^{k}}{k !} \sum_{i=0}^{k-2} \mathbf{Q}^{i}(-\mathbf{R}) \mathbf{Q}^{k-2-i} \mathbb{1} \frac{1}{E(\sigma)} \\
& =\int_{x=0}^{\infty} \sigma(x) \beta \underbrace{\sum_{k=2}^{\infty} \frac{x^{k}}{k !} \mathbf{Q}^{k-2}(\mathbf{Q}-\mathbb{1} \pi)^{2}}_{e^{\mathbf{Q}}-\mathbf{I}-x \mathbf{Q}+x^{2} \mathbb{1} \pi / 2}(\mathbf{Q}-\mathbb{1} \pi)^{-2}(-\mathbf{R}) \mathbb{1} \frac{1}{E(\sigma)} \\
& =\beta\left(\sigma^{*}(\mathbf{Q})-\mathbf{I}-E(\sigma) \mathbf{Q}\right)(\mathbf{Q}-\mathbb{1} \pi)^{-2}(-\mathbf{R}) \mathbb{1} \frac{1}{E(\sigma)}+\frac{E\left(\sigma^{2}\right)}{2 E(\sigma)} \pi(-\mathbf{R}) \mathbb{1} \\
& =\beta\left(\sigma^{*}(\mathbf{Q})-\mathbf{I}\right)(\mathbf{Q}-\mathbb{1} \pi)^{-2}(-\mathbf{R}) \mathbb{1} \frac{1}{E(\sigma)}-\beta \mathbf{Q}(\mathbf{Q}-\mathbb{1} \pi)^{-2}(-\mathbf{R}) \mathbb{1}-\lambda \frac{E\left(\sigma^{2}\right)}{2 E(\sigma)}
\end{aligned}
$$

The expression can be simplified further by making use of the identity $\mathbf{Q}(\mathbf{Q}-\mathbb{1} \pi)^{-2}=(\mathbf{Q}-\mathbb{1} \pi)^{-1}-\mathbb{1} \pi$ (see Lemma 6), resulting

$$
\lim _{s \rightarrow 0}\left(\frac{\mathrm{d}}{\mathrm{d} s} q_{1}^{*}(s)\right) q_{2}^{*}(s) \mathbb{1}=\beta\left(\sigma^{*}(\mathbf{Q})-\mathbf{I}\right)(\mathbf{Q}-\mathbb{1} \pi)^{-2}(-\mathbf{R}) \mathbb{1} \frac{1}{E(\sigma)}-\beta(\mathbf{Q}-\mathbb{1} \pi)^{-1}(-\mathbf{R}) \mathbb{1}+\lambda-\lambda E(\sigma) \frac{1+c_{\sigma}^{2}}{2}
$$

Let us now express and manipulate the second term of (74). Similar to (73), from Lemma 6 we have that

$$
\frac{\mathrm{d}}{\mathrm{d} s} q_{2}^{*}(s)=(\mathbf{R}-d \mathbf{I})^{-1}\left(\frac{(\mathbf{R}-d \mathbf{I}) \mathbb{1}}{\lambda-d} \pi-\mathbf{Q}(\mathbf{R}-d \mathbf{I})^{-1}\right)^{-1}-\frac{1}{d-\lambda} \mathbb{1} \pi,
$$

which, combined with (72) and observing that $\left(\sigma^{*}(\mathbf{Q})-\mathbf{I}\right)(\mathbf{Q}-\mathbb{1} \pi)^{-1} \mathbb{1}=0$ leads to

$$
\begin{aligned}
\lim _{s \rightarrow 0} q_{1}^{*}(s)\left(\frac{\mathrm{d}}{\mathrm{d} s} q_{2}^{*}(s) \mathbb{1}\right)=\underbrace{-E(\sigma) \frac{d}{c(d-\lambda)} \pi \mathbb{1}}_{-1} \\
\quad-\frac{d}{c} \beta\left(\sigma^{*}(\mathbf{Q})-\mathbf{I}\right)(\mathbf{Q}-\mathbb{1} \pi)^{-1}(\mathbf{R}-d \mathbf{I})^{-1}\left(\frac{(\mathbf{R}-d \mathbf{I}) \mathbb{1}}{\lambda-d} \pi-\mathbf{Q}(\mathbf{R}-d \mathbf{I})^{-1}\right)^{-1} \mathbb{1} \\
\quad-E(\sigma) \frac{d}{c} \pi(\mathbf{R}-d \mathbf{I})^{-1}\left(\frac{(\mathbf{R}-d \mathbf{I}) \mathbb{1}}{\lambda-d} \pi-\mathbf{Q}(\mathbf{R}-d \mathbf{I})^{-1}\right)^{-1} \mathbb{1} .
\end{aligned}
$$


Since $(\mathbb{1} \pi-\mathbf{Q})^{-1} \mathbf{Q}=\mathbb{1} \pi-\mathbf{I}$, we can write $\pi=\beta(\mathbb{1} \pi-\mathbf{Q})^{-1} \mathbf{Q}+\beta$ in the last term. Furthermore, in the second term we substitute $(\mathbf{Q}-\mathbb{1} \pi)^{-1}$ with $(\mathbf{Q}-\mathbb{1} \pi)^{-2} \mathbf{Q}-\mathbb{1} \pi$. These manipulations give

$$
\begin{aligned}
\lim _{s \rightarrow 0} q_{1}^{*}(s)\left(\frac{\mathrm{d}}{\mathrm{d} s} q_{2}^{*}(s) \mathbb{1}\right)=-1 \\
\quad-\frac{d}{c} \beta\left(\sigma^{*}(\mathbf{Q})-\mathbf{I}\right)(\mathbf{Q}-\mathbb{1} \pi)^{-2} \mathbf{Q}(\mathbf{R}-d \mathbf{I})^{-1}\left(\frac{(\mathbf{R}-d \mathbf{I}) \mathbb{1}}{\lambda-d} \pi-\mathbf{Q}(\mathbf{R}-d \mathbf{I})^{-1}\right)^{-1} \mathbb{1} \\
\quad-E(\sigma) \frac{d}{c} \beta(\mathbb{1} \pi-\mathbf{Q})^{-1} \mathbf{Q}(\mathbf{R}-d \mathbf{I})^{-1}\left(\frac{(\mathbf{R}-d \mathbf{I}) \mathbb{1}}{\lambda-d} \pi-\mathbf{Q}(\mathbf{R}-d \mathbf{I})^{-1}\right)^{-1} \mathbb{1} \\
\quad-E(\sigma) \frac{d}{c} \beta(\mathbf{R}-d \mathbf{I})^{-1}\left(\frac{(\mathbf{R}-d \mathbf{I}) \mathbb{1}}{\lambda-d} \pi-\mathbf{Q}(\mathbf{R}-d \mathbf{I})^{-1}\right)^{-1} \mathbb{1},
\end{aligned}
$$

since $\left(\sigma^{*}(\mathbf{Q})-\mathbf{I}\right) \mathbb{1}=0$. Utilizing that $E(\sigma) d / c=(d-\lambda)$ due to Lemma 2 , and that $\mathbf{Q}(\mathbf{R}-d \mathbf{I})^{-1}((\mathbf{R}-$ $\left.d \mathbf{I}) \mathbb{1} /(\lambda-d) \pi-\mathbf{Q}(\mathbf{R}-d \mathbf{I})^{-1}\right)^{-1} \mathbb{1}=-\mathbb{1}-(\mathbf{R}-d \mathbf{I}) \mathbb{1} /(d-\lambda)$, (79) further equals to

$$
\begin{aligned}
& \lim _{s \rightarrow 0} q_{1}^{*}(s)\left(\frac{\mathrm{d}}{\mathrm{d} s} q_{2}^{*}(s) \mathbb{1}\right)= \\
& \quad-E(\tilde{q})+\underbrace{\beta\left(\sigma^{*}(\mathbf{Q})-\mathbf{I}\right)(\mathbf{Q}-\mathbb{1} \pi)^{-2}(\mathbf{R}-d \mathbf{I}) \mathbb{1}}_{=\beta\left(\sigma^{*}(\mathbf{Q})-\mathbf{I}\right)(\mathbf{Q}-1 \pi)^{-2} \mathbf{R} \mathbb{1}} \frac{1}{E(\sigma)}+(d-\lambda)+\underbrace{\beta(\mathbb{1} \pi-\mathbf{Q})^{-1}(\mathbf{R}-d \mathbf{I}) \mathbb{1}}_{=\beta(\mathbb{1} \pi-\mathbf{Q})^{-1} \mathbf{R} \mathbb{1}-d},
\end{aligned}
$$

where we used the identity $(\mathbb{1} \pi-\mathbf{Q})^{-1} \mathbb{1}=\mathbb{1}$ several times. Adding (76) to (80) provides the theorem.

Theorem 9. $E(\tilde{q})$ is the mean length of the fluid queue without vacations, characterized by $(\mathbf{Q}, \mathbf{R}-d \mathbf{I})$.

Proof. Using the matrix-analytic approach, the density of the fluid level in a fluid model without vacations is given by $[16,9]$

$$
f(x)=p_{-} \tilde{\mathbf{Q}}^{-+} e^{\mathbf{K} x}\left[\begin{array}{ll}
\mathbf{I} & \mathbf{\Psi}
\end{array}\right]|\mathbf{R}-d \mathbf{I}|^{-1},
$$

where $p_{-}$is the probability mass vector corresponding to level 0 , which, in our case, equals to $p_{-}=(d-\lambda) \omega$. The LST of the fluid level is

$$
f^{*}(s)=(d-\lambda) \omega \tilde{\mathbf{Q}}^{-+}(s \mathbf{I}-\mathbf{K})^{-1}\left[\begin{array}{ll}
\mathbf{I} & \mathbf{\Psi}
\end{array}\right]|\mathbf{R}-d \mathbf{I}|^{-1} .
$$

Using the results of Lemma 5 it can be transformed to

$$
f^{*}(s)=(d-\lambda) \omega \tilde{\mathbf{Q}}^{-+}\left[\begin{array}{ll}
\mathbf{I} & \mathbf{\Psi}
\end{array}\right]|\mathbf{R}-d \mathbf{I}|^{-1}(\mathbf{R}-d \mathbf{I})(s(\mathbf{R}-d \mathbf{I})-\mathbf{Q})^{-1} .
$$

Investigating the block structure of the matrix in the middle gives

$$
\begin{aligned}
\tilde{\mathbf{Q}}^{-+}\left[\begin{array}{rr}
\mathbf{I} & \mathbf{\Psi}
\end{array}\right]|\mathbf{R}-d \mathbf{I}|^{-1}(\mathbf{R}-d \mathbf{I}) & =\left[\begin{array}{ll}
-\left(\mathbf{R}^{-}-d \mathbf{I}\right)^{-1} \mathbf{Q}^{-+} & \left(\mathbf{R}^{-}-d \mathbf{I}\right)^{-1} \mathbf{Q}^{-+} \mathbf{\Psi}
\end{array}\right] \\
& =\left[\begin{array}{ll}
-\left(\mathbf{R}^{-}-d \mathbf{I}\right)^{-1} \mathbf{Q}^{-+} & -\mathbf{U}-\left(\mathbf{R}^{-}-d \mathbf{I}\right)^{-1} \mathbf{Q}^{--}
\end{array}\right]
\end{aligned}
$$

leading to

$$
f^{*}(s)=(d-\lambda) \omega\left(-\left(\mathbf{R}^{-}-d \mathbf{I}\right)^{-1}\right)\left[\mathbf{Q}^{-+} \quad \mathbf{Q}^{--}\right](s(\mathbf{R}-d \mathbf{I})-\mathbf{Q})^{-1},
$$

since $\omega \mathbf{U}=0$. Introducing $\beta$ as an initial vector makes it possible to introduce the positive block row of $\mathbf{Q}$ as well, since $\beta^{+}=0$. Finally, the LST of the fluid level is

$$
\begin{aligned}
f^{*}(s) & =-(d-\lambda) \beta(\mathbf{R}-d \mathbf{I})^{-1} \mathbf{Q}(s(\mathbf{R}-d \mathbf{I})-\mathbf{Q})^{-1} \\
& =-(d-\lambda) \beta(\mathbf{R}-d \mathbf{I})^{-1} \mathbf{Q}(\mathbf{R}-d \mathbf{I})^{-1}\left(s \mathbf{I}-\mathbf{Q}(\mathbf{R}-d \mathbf{I})^{-1}\right)^{-1} .
\end{aligned}
$$

Taking the derivative of the LST and applying Lemma 6 with $\mathbf{X}=\mathbf{Q}(\mathbf{R}-d \mathbf{I})^{-1}, x_{l}=\pi$ and $x_{r}^{T}=$ $(\mathbf{R}-d \mathbf{I}) \mathbb{1} /(\lambda-d)$ proves the theorem. 


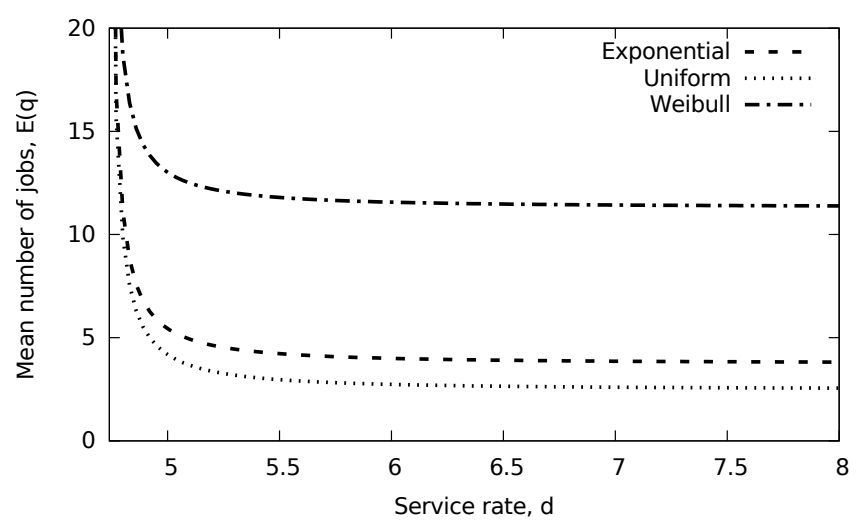

Figure 1: Mean fluid level as the function of the service rate

\section{Numerical example}

The main results of the paper, the mean fluid level formulas provided by Theorem 7 and 8 are easy to implement. The computationally most challenging step is the calculation of initial vector $\beta$, that involves the solution of a NARE. Most mathematical frameworks do not include a built-in solution for NAREs, but there are some efficient algorithms available in the literature that are able to cope with large matrices (up to several thousand rows and columns) without any numerical problems. In the following example we used the cyclic reduction algorithm published in [18].

The generator of the background process and the rates associated with the states in the example are

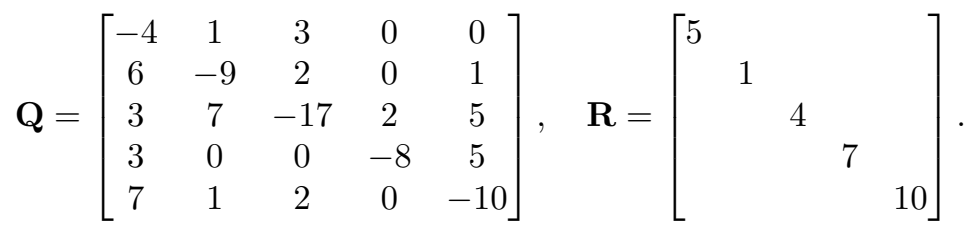

For the distribution of the vacation period let us consider three cases:

- Exponential distribution with mean $E(\sigma)=0.8$. The squared coefficient of variation is $c_{\sigma}^{2}=1$.

- Uniform distribution between 0 and 1.6 , thus $E(\sigma)=0.8, c_{\sigma}^{2}=1 / 3$.

- Weibull distribution with scale parameter $1 / 2$ and scale parameter 0.4 , providing $E(\sigma)=0.8, c_{\sigma}^{2}=5$.

With these parameters the mean arrival rate is $\lambda=4.73$, thus for the service rate $d>4.73$ must hold for stability.

Figure 1 depicts the mean fluid level in the system as the function of the service rate. As expected, the mean fluid level is decreasing if the service rate is increasing. As a consequence of Theorem 8, the mean fluid level depends only on the first two moments of the distribution of the vacation period. The higher the squared coefficient of variation of the vacation period is, the higher is the mean fluid level. The state dependent mean fluid level is depicted in Figure 2 in the case when the vacation period is uniformly distributed.

\section{References}

[1] B. T. Doshi, Queueing systems with vacations - a survey, Queueing Systems 1 (1986) 29-66.

[2] H. Takagi, Queuing Analysis of Polling Models, ACM Comput. Surv. 20 (1) (1988) 5-28.

[3] R. Carsten, E. Newhall, M. Posner, A Simplified Analysis of Scan Times in an Asymmetrical Newhall Loop with Exhaustive Service, Communications, IEEE Transactions on 25 (9) (1977) 951-957. 


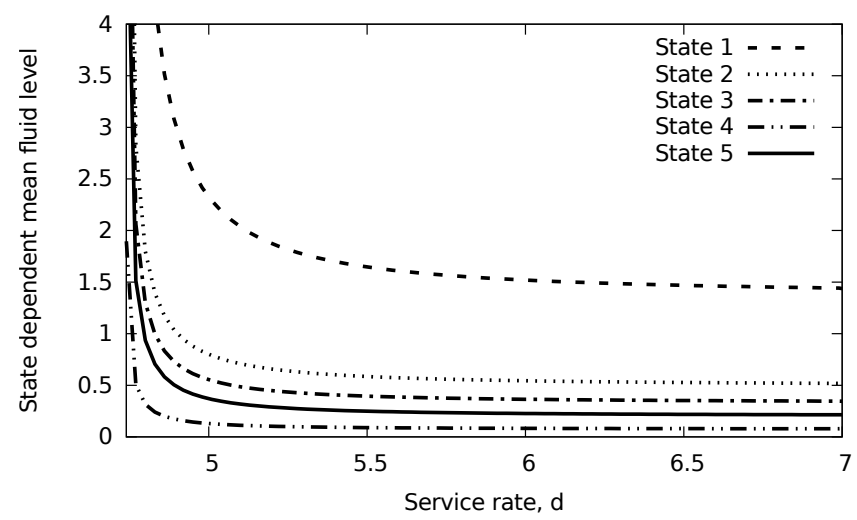

Figure 2: The state-dependent mean fluid level as the function of the service rate

[4] U. S. Karmarkar, Lot Sizes, Lead Times and In-Process Inventories, Management Science 33 (3) (1987) 409-418.

[5] S. W. Fuhrmann, R. B. Cooper, Stochastic Decompositions in the M/G/1 Queue with Generalized Vacations, Operations Research 33 (1985) 1117-1129.

[6] D. Mitra, Stochastic Theory of a Fluid Model of Producers and Consumers Coupled by a Buffer, Advances in Applied Probability 20 (3) (1988) pp. 646-676.

[7] V. G. Kulkarni, Fluid Models for Single Buffer Systems, in: J. H. Dshalalow (Ed.), Frontiers in Queueing, CRC Press, Inc., Boca Raton, FL, USA, ISBN 0-8493-8076-6, 321-338, 1997.

[8] S. Ahn, V. Ramaswami, Efficient Algorithms for Transient Analysis of Stochastic Fluid Flow Models, Journal of Applied Probability 42 (2) (2005) pp. 531-549.

[9] A. da Silva Soares, G. Latouche, Matrix-analytic methods for fluid queues with finite buffers, Performance Evaluation $63(4-5)(2006) 295-314$.

[10] D. Anick, D. Mitra, M. M. Sondhi, Stochastic Theory of a Data-Handling System with Multiple Sources, Bell System Technical Journal 61 (8) (1982) 1871-1894.

[11] O. Czerniak, U. Yechiali, Fluid polling systems, Queueing System 63 (2009) 401-435.

[12] Z. Saffer, M. Telek, Fluid vacation model with Markov modulated load and gated discipline, in: 9th International Conference on Queueing and Network Applications (QTNA), 184-197, 2014.

[13] Z. Saffer, M. Telek, Fluid vacation model with Markov modulated load and exhaustive discipline, in: Computer Performance Engineering, EPEW, vol. 8721 of $L N C S, 59-73,2014$.

[14] B. Sericola, M.-A. Remiche, Maximum Level and Hitting Probabilities in Stochastic Fluid Flows Using Matrix Differential Riccati Equations, Methodology and Computing in Applied Probability 13 (2)

[15] A. Reibman, R. Smith, K. Trivedi, Markov and Markov reward model transient analysis: an overview of numerical approaches, European Journal of Operational Research 40 (1989) 257-267.

[16] A. D. S. Soares, Fluid queues - building upon the analysis with QBD processes, Ph.D. thesis, Universit Libre de Bruxelles, 2005.

[17] S. Ahn, V. Ramaswami, Efficient algorithms for transient analysis of stochastic fluid flow models, Journal of Applied Probability (2005) 531-549.

[18] D. A. Bini, B. Iannazzo, G. Latouche, B. Meini, On the solution of algebraic Riccati equations arising in fluid queues, Linear Algebra and its applications 413 (2) (2006) 474-494. 